\title{
Hydrogen- and Halogen-Bonded Binary Cocrystals with Ditopic Components: Systematic Structural and Photoreactivity Properties that Provide Access to a Series of Symmetrical Cyclobutanes
}

Jay Quentin ${ }^{\dagger}$ and Leonard R. MacGillivray*, ${ }^{\dagger}$

${ }^{\dagger}$ Department of Chemistry, University of Iowa, Department of Chemistry, Iowa City, IA 52242, United States

*Corresponding Author:

Leonard R. MacGillivray

E-mail: len-macgillivray@uiowa.edu

ORCID

Jay Quentin: 0000-0002-1729-7774

Leonard R. MacGillivray: 0000-0003-0875-677X 


\section{Supporting Information:}

S1. Synthetic Procedures

trans-1,2-bis(3-pyridyl)ethylene (3,3'-bpe).

(res) $\cdot(2,2$ '-bpe).

(1,3-di-I-tFb) (3,3'-bpe).

4(res) $3(3,3$ '-bpe).

(1,3-di-I-tFb) $(4,4$ '-bpe).

(res) $\cdot(4,4$ '-bpe).

$3(\mathbf{h q}) \cdot 2\left(2,2^{\prime}\right.$-bpe).

(1,4-di-I-tFb) (3,3'-bpe).

(hq) $\cdot(3,3$ '-bpe).

(1,4-di-I-tFb) (4,4'-bpe).

(hq) $\cdot(4,4$ '-bpe $)$.

S2. Proton Nuclear Magnetic Resonance $\left({ }^{1} \mathrm{H}\right.$ NMR) Spectroscopy Data

Figure S1. ${ }^{1} \mathrm{H}$ NMR spectrum of 4(res)'(3,3'-bpe).

Figure S2. ${ }^{1} \mathrm{H}$ NMR spectrum of $3(\mathbf{h q}) \cdot 2$ (2,2'-bpe).

Figure S3. ${ }^{1} \mathrm{H}$ NMR spectrum of (hq)·(3,3'-bpe).

S3. Powder X-Ray Diffraction (pXRD) Data

Figure S4. pXRD pattern of (res) (2,2'-bpe).

Figure S5. pXRD pattern of (1,3-di-I-tFb) (3,3'-bpe).

Figure S6. pXRD pattern of 4(res)·3(3,3'-bpe).

Figure S7. pXRD pattern of (1,3-di-I-tFb) (4,4'-bpe).

Figure S8. pXRD pattern of (res) (4,4'-bpe)

Figure S9. pXRD pattern of 3(hq) 2 (2,2'-bpe).

Figure S10. pXRD pattern of (1,4-di-I-tFb) (3,3’-bpe).

Figure S11. pXRD pattern of (hq) (3,3'-bpe).

Figure S12. pXRD pattern of (1,4-di-I-tFb) (4,4'-bpe).

Figure S13. pXRD pattern of (hq)·(4,4'-bpe).

S4. Single-crystal X-Ray Diffraction (scXRD) Data

Table S1. Crystallographic data and structure refinement statistics for (1,3-di-I-tFb)·(3,3'-bpe).

Table S2. Crystallographic data and structure refinement statistics for 4(res) $3(3,3$ '-bpe).

Table S3. Crystallographic data and structure refinement statistics for (1,3-di-I-tFb)·(4,4'-bpe).

Table S4. Crystallographic data and structure refinement statistics for 3(hq)·2(2,2'-bpe).

Table S5. Crystallographic data and structure refinement statistics for (1,4-di-I-tFb)·(3,3'-bpe).

Table S6. Crystallographic data and structure refinement statistics for (hq)·(3,3'-bpe).

S5. Bond Metrics and Melting Point Tables

Table S7. Hydrogen bond metrics for H-bonded cocrystals.

Table S8. Bond metrics for X-bonded cocrystals.

Table S9. Melting point data for H-bonded cocrystals and coformers thereof.

Table S10. Melting point data for X-bonded cocrystals and coformers thereof.

S7. References 


\section{S1. Synthetic Procedures}

Materials and Methods. All reagents and solvents (synthesis grade) were purchased from commercial sources and used as received unless otherwise stated. Triethoxyvinylsilane, 3-bromopyridine, palladium(II) acetate, resorcinol (res), hydroquinone (hq), 1,4-diiodotetrafluorobenzene (1,4-di-I-tFb), and trans-1,2-bis(4pyridyl)ethylene (4,4'-bpe) were purchased from Aldrich ${ }^{\odot}$; trans-1,2-bis(2-pyridyl)ethylene (2,2'-bpe) was purchased from TCI $^{\odot} ;$ 1,3-diiodotetrafluorobenzene (1,3-di-I-tFb) was purchased from Apollo Scientific ${ }^{\odot}$. All cocrystal syntheses were conducted in screw-cap glass scintillation vials. For cocrystal syntheses, "thermal dissolution' refers to the process of: combining both solid cocrystal components in a vial; adding solvent portionwise while maintaining a saturated mixture at rt; tightly capping the vial and heating the mixture on a hot-plate until all solids dissolve to afford a homogeneous solution with the minimum necessary volume of solvent. Compositions of all single crystals were shown to be representative of the bulk material by matching experimental pXRD patterns with those simulated from scXRD data. Yields refer to isolated yields of analytically pure compounds unless otherwise stated. Thin-layer chromatography (TLC) assay employed polyester backed SORBTECH (Sorbent Technologies, Inc.) Silica G TLC plates with UV254 fluorescent indicator (200 $\mu \mathrm{m}$ sorbent thickness). TLC visualization was accomplished by a successive three-step sequence employing short-wave (254 $\mathrm{nm}$ ) and long-wave (365 nm) UV light, $\mathrm{I}_{2}$ vapor, and $\mathrm{KMnO}_{4(\mathrm{aq})}$. Flash chromatography columns were packed with 230-400 mesh SiliaFlash ${ }^{\circledR}$ F60 silica gel (SiliCycle, Inc. ${ }^{\odot}$ ) as a slurry in the eluant. Crude product mixtures purified by FC were loaded onto the column as solutions in the eluant. Melting points were recorded on samples in open capillary tubes using a manual MEL-TEMP ${ }^{\circledR}$ apparatus (Electrothermal ${ }^{\mathrm{TM}}$ Corporation) and are uncorrected. Photoactivities of the cocrystals were assessed by exposing each solid to UV-radiation for a period of30 hours. UV-irradiations were conducted in an $\mathrm{ACE}^{\circledR}$ photocabinet equipped with an $\mathrm{ACE}^{\circledR}$ quartz, $450 \mathrm{~W}$, broadband, ${ }^{*}$ medium pressure, Hg-vapor lamp. Samples were irradiated by: 1) grinding single crystals of the cocrystal to a fine powder with an agate mortar and pestle; 2) smearing the powder between two Pyrex ${ }^{\circledR}$ plates; 3) irradiating the powder in $10 \mathrm{~h}$ intervals, taking care to ensure uniform irradiation. ${ }^{\dagger}$

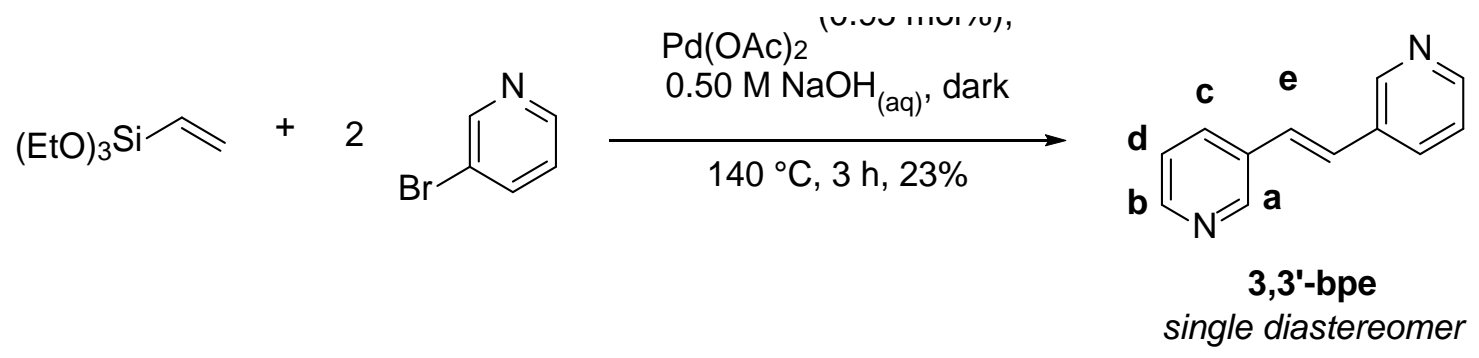

trans-1,2-bis(3-pyridyl)ethylene (3,3'-bpe). The compound was prepared as previously described. ${ }^{1}$ All reaction steps were conducted in the dark. A 35-mL ACE ${ }^{\circledR}$ pressure tube equipped with a magnetic stir-bar was charged with triethoxyvinylsilane $(1.17 \mathrm{~mL}, 5.38 \mathrm{mmol})$ and $0.50 \mathrm{M} \mathrm{NaOH}_{(\mathrm{aq})}(13.5 \mathrm{~mL}, 6.75 \mathrm{mmol}, 1.25$ equiv based on triethoxyvinylsilane). The biphasic mixture was stirred vigorously at rt for $22 \mathrm{~min}$, and 3-bromopyridine (1.10 mL, 11.3 mmol, 2.10 equiv) and $\mathrm{Pd}(\mathrm{OAc})_{2}$ (11.7 mg, 5.1 x 10 ${ }^{-2} \mathrm{mmol}, 0.95 \mathrm{~mol} \%$ ) were successively added. The tube was sealed and immersed in an oil bath-preheated to $140{ }^{\circ} \mathrm{C}$ - and stirred vigorously. After $3 \mathrm{~h}$, the mixture was allowed to cool to rt and diluted with EtOAc $(80 \mathrm{~mL})$ and $\mathrm{H}_{2} \mathrm{O}(80 \mathrm{~mL})$. The layers were separated and the aqueous phase extracted with EtOAc $(3 \times 20 \mathrm{~mL})$. Combined organic phases were washed with brine $(1 \times 100$ $\mathrm{mL}$ ), dried $\left(\mathrm{Na}_{2} \mathrm{SO}_{4}\right)$, and filtered through Celite ${ }^{\circledR} 545$, employing EtOAc to rinse the filter cake. Concentration in vacuo followed by drying under high vacuum afforded a yellow-orange oil. None of the [undesired] cis-isomer

${ }^{*} \lambda=1367.3-222.4 \mathrm{~nm}$. Of the total energy emitted by the broadband lamp, approximately $40-48 \%$ is in the ultraviolet portion of the spectrum, $40-43 \%$ in the visible, and the balance in the infrared.

${ }^{\dagger}$ Uniform irradiation of cocrystal powders was ensured by: occasionally scraping (razor blade) the powder from each plate assembly; combining powders from all plate assemblies; homogenizing the combined, bulk powder via thorough grinding (agate mortar and pestle); redistributing the homogenized powder among each plate assembly. Each plate assembly was also flipped between irradiation intervals to ensure equal irradiation of both faces of the plate assembly. 
was detectable by ${ }^{1} \mathrm{H}$ NMR $\left(400 \mathrm{MHz}, \mathrm{CDCl}_{3}\right)$ assay of the crude solid. ${ }^{\ddagger}$ Flash chromatography $\left(\mathrm{SiO}_{2}, 10 \%\right.$ $\mathrm{MeOH} / \mathrm{CH}_{2} \mathrm{Cl}_{2}$ ) furnished 3,3'-bpe as yellow crystals: $222.3 \mathrm{mg}(23 \%)$. Analytical data: ${ }^{1} \mathrm{H} \mathrm{NMR}(400 \mathrm{MHz}$, $\left.\mathrm{CDCl}_{3}\right) \delta 8.74\left(\mathrm{~d}, J=1.7 \mathrm{~Hz}, 2 \mathrm{H}_{\mathrm{a}}\right.$ ), 8.53 (dd, $\left.J=4.8,1.5 \mathrm{~Hz}, 2 \mathrm{H}_{\mathrm{b}}\right), 7.86$ (app dt, $J=8.0,1.9 \mathrm{~Hz}, 2 \mathrm{H}_{\mathrm{c}}$ ), 7.32 (ddd, $\left.J=7.9,4.8,0.8 \mathrm{~Hz}, 2 \mathrm{H}_{\mathrm{d}}\right), 7.14$ (s, $\left.2 \mathrm{H}_{\mathrm{e}}\right)$. TLC $\left(\mathrm{SiO}_{2}, 10 \% \mathrm{MeOH} / \mathrm{CH}_{2} \mathrm{Cl}_{2}\right): R_{f}=0.35$. A portion of 3,3'-bpe was dissolved in $\mathrm{CHCl}_{3}$ and allowed to slowly evaporate: single crystals - colorless plates, suitable for scXRDformed within $7 \mathrm{~d}$.

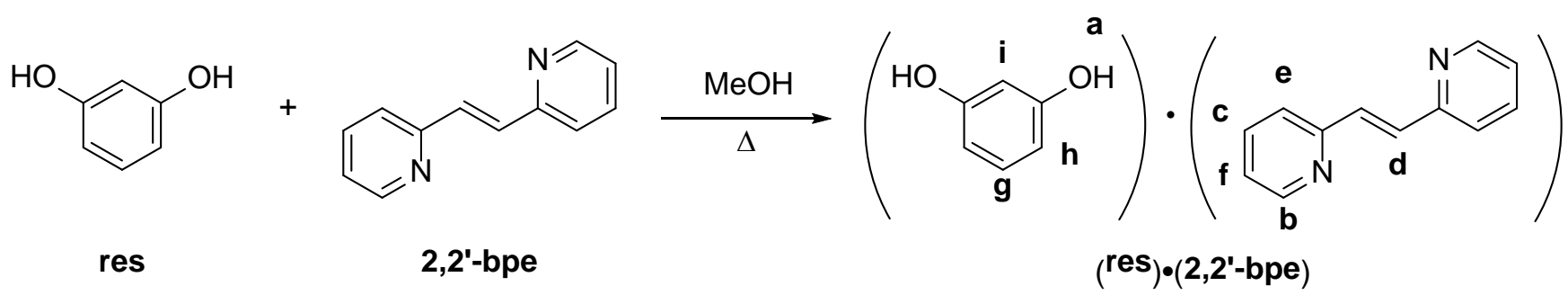

(res)·(2,2'-bpe). Note: (res)'(2,2'-bpe) is photosensitive. Cocrystals of (res)·(2,2'-bpe) were obtained by thermal dissolution of 2,2'-bpe (313.1 mg, $1.68 \mathrm{mmol}$ ) and res (183.7 mg, $1.65 \mathrm{mmol}, 0.981$ equiv) in $\mathrm{MeOH}$ (5.0 mL). Upon cooling to rt, single crystals of (res) (2,2'-bpe) formed within $36 \mathrm{~d}$. Analytical data: $\mathbf{m p} 95-97^{\circ} \mathrm{C}(\mathrm{MeOH})$. ${ }^{1} \mathbf{H}$ NMR (400 MHz, DMSO- $d_{6}$ ): $\delta 9.18\left(\mathrm{~s}, 2 \mathrm{H}_{\mathrm{a}}\right.$ ), 8.60 (ddd, $J=4.8,1.8,0.8 \mathrm{~Hz}, 2 \mathrm{H}_{\mathrm{b}}$ ), 7.81 (app td, $J=7.7,1.8$ $\mathrm{Hz}, 2 \mathrm{H}_{\mathrm{c}}$ ), 7.69 (s, 2H $\mathrm{H}_{\mathrm{d}}$ ), 7.63 (td, $J=7.8,1.0 \mathrm{~Hz}, 2 \mathrm{H}_{\mathrm{e}}$ ), 7.30 (ddd, $J=7.5,4.8,1.1 \mathrm{~Hz}, 2 \mathrm{H}_{\mathrm{f}}$ ), 6.93-6.88 (m, $1 \mathrm{H}_{\mathrm{g}}$ ), 6.19-6.18 (m, $\left.2 \mathrm{H}_{\mathrm{h}}\right), 6.17\left(\mathrm{~m}, 1 \mathrm{H}_{\mathrm{i}}\right)$.<smiles>Fc1c(F)c(I)c(I)c(F)c1I</smiles>

1,3-di-I-tFb<smiles>C(=C/c1cccnc1)\c1cccnc1</smiles>

3,3'-bpe

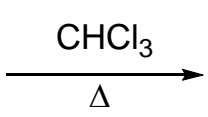<smiles>Fc1c(F)c(I)c(I)c(I)c1I</smiles>

(1,3-di-I-tFb)·(3,3'-bpe). Cocrystals of (1,3-di-I-tFb)·(3,3'-bpe) were obtained by thermal dissolution of 3,3'bpe $(64.5 \mathrm{mg}, 0.354 \mathrm{mmol})$ and 1,3-di-I-tFb (153.0 mg, $0.369 \mathrm{mmol}, 1.04$ equiv) in $\mathrm{CHCl}_{3}(1.5 \mathrm{~mL})$. Upon cooling to rt, single crystals of (1,3-di-I-tFb)·(3,3'-bpe)—colorless, irregular prisms, suitable for scXRDformed within $5 \mathrm{~d}$. Analytical data: $\mathbf{m p} 102-105^{\circ} \mathrm{C}$ (dec., $\mathrm{CHCl}_{3}$ ).

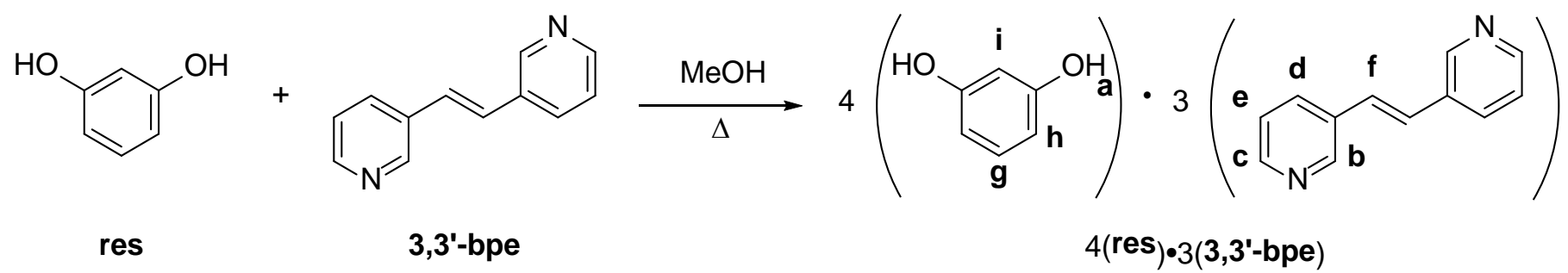

4(res)·3(3,3'-bpe). Cocrystals of 4(res)·3(3,3'-bpe) were obtained by thermal dissolution of 3,3'-bpe (42.6 mg, $0.234 \mathrm{mmol}$ ) and res (26.5 mg, $0.238 \mathrm{mmol}, 1.02$ equiv) in $\mathrm{MeOH}$ (1.5 mL). Upon cooling to rt, single crystals of 4(res)·3(3,3'-bpe) — colorless plates, suitable for scXRD—formed within $3 \mathrm{~d}$. Analytical data: $\mathbf{m p} 122-130{ }^{\circ} \mathrm{C}$

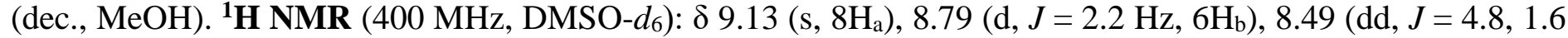
$\mathrm{Hz}, 6 \mathrm{H}_{\mathrm{c}}$ ), 8.07 (app dt, $J=8.0,1.9 \mathrm{~Hz}, 6 \mathrm{H}_{\mathrm{d}}$ ), 7.43 (dd, $J=7.8,4.8 \mathrm{~Hz}, 6 \mathrm{H}_{\mathrm{e}}$, overlapped with $\mathrm{H}_{\mathrm{f}}$ ), 7.43 (s, $6 \mathrm{H}_{\mathrm{f}}$, overlapped with $\left.\mathrm{H}_{\mathrm{e}}\right)$, 6.92-6.88 (m, $\left.4 \mathrm{H}_{\mathrm{g}}\right), 6.19-6.18\left(\mathrm{~m}, 8 \mathrm{H}_{\mathrm{h}}\right), 6.17-6.16\left(\mathrm{~m}, 4 \mathrm{H}_{\mathrm{i}}\right)$.

${ }^{\ddagger}$ The cis-isomer of 3,3’-bpe was not observed when referencing previously reported ${ }^{1} \mathrm{H}$ NMR spectral data for that isomer. See: S. P. Gromov, A. I. Vedernikov, E. N. Ushakov, N. A. Lobova, A. A. Botsmanova, L. G. Kuz'mina, A. V. Churakov, Y. A. Strelenko, M. V. Alfimov, J. A. K. Howard, D. Johnelsf , U. G. Edlund, New J. Chem. 2005, 29, 881-894. 
$\overbrace{F}^{(}$

1,3-di-I-tfb<smiles>C(=C/c1ccncc1)\c1ccncc1</smiles>

4,4'-bpe

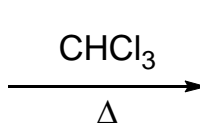

$(\overbrace{F}^{(}$

(1,3-di-I-tFb)·(4,4'-bpe). Cocrystals of (1,3-di-I-tFb)·(4,4'-bpe) were obtained by thermal dissolution of 4,4'bpe $(82.2 \mathrm{mg}, 0.438 \mathrm{mmol})$ and 1,3-di-I-tFb (175.0 mg, $0.422 \mathrm{mmol}, 0.97$ equiv) in $\mathrm{CHCl}_{3}(2.0 \mathrm{~mL})$. Upon cooling to rt, single crystals of (1,3-di-I-tFb) (4,4'-bpe) — colorless plates, suitable for scXRD—formed within 103 min. Analytical data: mp $159^{\circ} \mathrm{C}$ (dec., $\left.\mathrm{CHCl}_{3}\right)$.<smiles>Oc1cccc(O)c1</smiles>

res<smiles>C(=C/c1ccncc1)\c1ccncc1</smiles>

4,4'-bpe
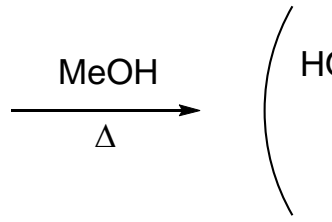<smiles>Cl/C=C/c1ccncc1</smiles>

$\left({ }^{\text {res }}\right) \cdot(4,4$ '-bpe $)$

(res)·(4,4'-bpe). Note: (res) (4,4'-bpe) is photosensitive. Cocrystals of (res)·(4,4'-bpe) were obtained by thermal dissolution of 4,4'-bpe (344.9 mg, $1.84 \mathrm{mmol}$ ) and res (203.2 mg, $1.83 \mathrm{mmol}, 1.00$ equiv) in $\mathrm{MeOH}(6.0 \mathrm{~mL})$. Upon cooling to rt, single crystals of (res)·(4,4'-bpe)—yellow blocks, suitable for scXRD_formed within $19 \mathrm{~h}$. Analytical data: mp $217{ }^{\circ} \mathrm{C}$ (dec., MeOH). ${ }^{1} \mathbf{H}$ NMR (400 MHz, DMSO-d 6 ): $\delta 9.13$ (s, $2 \mathrm{H}_{\mathrm{a}}$ ), 8.60 (dd, $J=4.5$, $\left.1.6 \mathrm{~Hz}, 4 \mathrm{H}_{\mathrm{b}}\right), 7.61$ (dd, $\left.J=4.5,1.6 \mathrm{~Hz}, 4 \mathrm{H}_{\mathrm{c}}\right), 7.54$ (s, 2H $\mathrm{H}_{\mathrm{d}}, 6.93-6.88\left(\mathrm{~m}, 1 \mathrm{H}_{\mathrm{e}}\right), 6.18\left(\mathrm{~m}, 2 \mathrm{H}_{\mathrm{f}}\right), 6.17-6.16\left(\mathrm{~m}, 1 \mathrm{H}_{\mathrm{g}}\right)$.

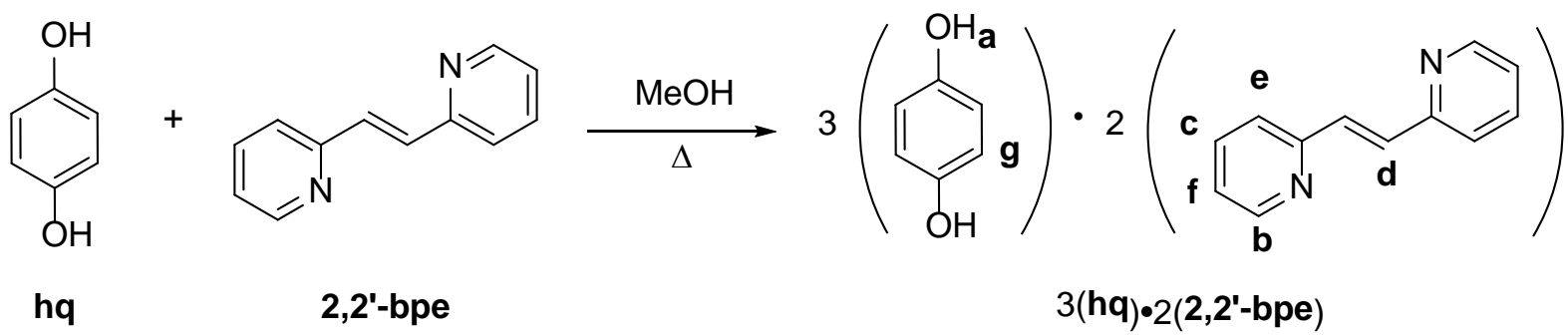

3(hq)·2(2,2'-bpe). Cocrystals of 3(hq)·2(2,2'-bpe) were obtained by thermal dissolution of 2,2'-bpe (149.4 mg, $0.795 \mathrm{mmol}$ ) and hq (88.9 mg, $0.799 \mathrm{mmol}, 1.01 \mathrm{equiv})$ in $\mathrm{MeOH}$ (2.0 mL). Upon cooling to rt, single crystals of 3(hq)·2(3,3'-bpe) — colorless prisms, suitable for scXRD—formed within 69 h. Analytical data: mp 119-128 ${ }^{\circ} \mathrm{C}$ (dec., MeOH). ${ }^{1} \mathbf{H}$ NMR (400 MHz, DMSO- $\left.d_{6}\right): \delta 8.61$ (br s, $6 \mathrm{H}_{\mathrm{a}}$ [overlapped with $\mathrm{H}_{\mathrm{b}}$ ]), 8.61-8.60 (m, $4 \mathrm{H}_{\mathrm{b}}$ [overlapped with $\mathrm{H}_{\mathrm{a}}$ ]), 7.81 (app td, $J=7.7,1.8 \mathrm{~Hz}, 4 \mathrm{H}_{\mathrm{c}}$ ), 7.70 (s, 4H $\mathrm{H}_{\mathrm{d}}, 7.63$ (d, $J=7.8 \mathrm{~Hz}, 4 \mathrm{He}$ ), 7.30 (ddd, $J=$ 7.5, 4.8, $\left.1.1 \mathrm{~Hz}, 4 \mathrm{H}_{\mathrm{f}}\right), 6.55$ (s, $\left.12 \mathrm{H}_{\mathrm{g}}\right)$.<smiles>Cc1c(F)c(F)c(I)c(F)c1F</smiles>

1,4-di-I-tfb<smiles>C(=C/c1cccnc1)\c1cccnc1</smiles>

3,3'-bpe

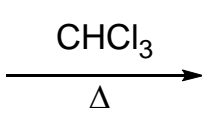<smiles>Cc1cccnc1</smiles>

(1,4-di-I-tfb)•(3,3'-bpe)

(1,4-di-I-tFb)·(3,3'-bpe). Cocrystals of (1,4-di-I-tFb)·(3,3'-bpe) were obtained by thermal dissolution of 3,3'bpe (48.2 mg, $0.265 \mathrm{mmol}$ ) and 1,4-di-I-tFb (109.3 mg, $0.267 \mathrm{mmol}, 1.01$ equiv) in $\mathrm{CHCl}_{3}(1.5 \mathrm{~mL})$. Upon cooling to rt, single crystals of (1,4-di-I-tFb)·(3,3'-bpe)—colorless, irregular prisms, suitable for scXRDformed within 20 h. Analytical data: mp $138-140{ }^{\circ} \mathrm{C}$ (dec., $\mathrm{CHCl}_{3}$ ). 
OH

hq<smiles>C(=C/c1cccnc1)\c1cccnc1</smiles>

3,3'-bpe<smiles>COc1ccc(O)cc1C=Cc1cccnc1</smiles>

(hq) $\cdot\left(3,3^{\prime}-\right.$ bpe $)$

(hq)·(3,3'-bpe). Cocrystals of (hq)·(3,3'-bpe) were obtained by slow evaporation of a solution of 3,3'-bpe (62.1 mg, $0.341 \mathrm{mmol})$ and hq (37.7 mg, $0.340 \mathrm{mmol}, 0.99$ equiv) in $\mathrm{MeOH}(7.0 \mathrm{~mL})$. Single crystals of (hq) (3,3’bpe)—colorless blocks, suitable for scXRD_formed within $11 \mathrm{~d}$. Analytical data: $\mathbf{m p ~} 167-171{ }^{\circ} \mathrm{C}(\mathrm{MeOH}) .{ }^{1} \mathbf{H}$ NMR (400 MHz, DMSO- $d_{6}$ ): $\delta 8.79$ (d, $J=2.2 \mathrm{~Hz}, 2 \mathrm{H}_{\mathrm{a}}$ ), 8.60 (s, 2H $\left.\mathrm{H}_{\mathrm{b}}\right), 8.49$ (dd, $J=4.7,1.6 \mathrm{~Hz}, 2 \mathrm{H}_{\mathrm{c}}$ ), 8.07 (app dt, $\left.J=8.0,1.9 \mathrm{~Hz}, 2 \mathrm{H}_{\mathrm{d}}\right), 7.44-7.41\left(\mathrm{~m}, 2 \mathrm{H}_{\mathrm{e}}\right.$ [overlapped with $\mathrm{H}_{\mathrm{f}}$ ]), 7.43 (s, $2 \mathrm{H}_{\mathrm{f}}$ [overlapped with $\mathrm{H}_{\mathrm{e}}$ ]).<smiles>Cc1c(F)c(F)c(I)c(F)c1F</smiles>

1,4-di-I-tfb<smiles>C(=Cc1cccnc1)c1ccncc1</smiles>

4,4'-bpe

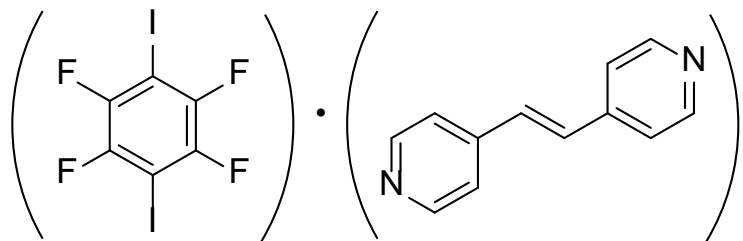

(1,4-di-I-tfb)•(4,4'-bpe)

(1,4-di-I-tFb)•(4,4'-bpe). Cocrystals of (1,4-di-I-tFb)·(4,4'-bpe) were obtained by thermal dissolution of 4,4'bpe (121.2 mg, $0.2645 \mathrm{mmol})$ and 1,4-di-I-tFb (267.0 mg, $0.651 \mathrm{mmol}, 1.01$ equiv) in $\mathrm{CHCl}_{3}(10.0 \mathrm{~mL})$. Upon cooling to rt, single crystals of (1,4-di-I-tFb) $(\mathbf{4 , 4}$ '-bpe) — pale-yellow plates, suitable for scXRD—formed within 14 h. Analytical data: $\mathbf{m p}>260{ }^{\circ} \mathrm{C}$ (dec., $\mathrm{CHCl}_{3}$ ).<smiles>Oc1ccc(O)cc1</smiles>

hq

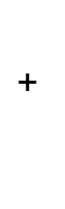<smiles>C(=C/c1ccncc1)\c1ccncc1</smiles>

4,4'-bpe

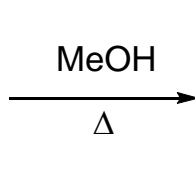<smiles>OCc1cc(O)ccc1O</smiles>

\section{(hq) $\bullet(4,4$ '-bpe $)$}

(hq)・(4,4'-bpe). Cocrystals of (hq) (4,4'-bpe) were obtained by thermal dissolution of 4,4'-bpe (121.7 mg, 0.648 $\mathrm{mmol}$ ) and hq (73.0 mg, $0.656 \mathrm{mmol}, 1.01$ equiv) in $\mathrm{MeOH}(4.0 \mathrm{~mL})$. Upon cooling to rt, single crystals of (hq) (4,4'-bpe) formed within $22 \mathrm{~h}$. Analytical data: mp $224-225^{\circ} \mathrm{C}$ (dec., MeOH). ${ }^{\mathbf{1}} \mathbf{H}$ NMR (400 MHz, DMSO$\left.d_{6}\right): \delta 8.64\left(\mathrm{~s}, 2 \mathrm{H}_{\mathrm{a}}\right), 8.59$ (dd, $\left.J=4.5,1.5 \mathrm{~Hz}, 4 \mathrm{H}_{\mathrm{b}}\right), 7.61$ (dd, $\left.J=4.6,1.6 \mathrm{~Hz}, 4 \mathrm{H}_{\mathrm{c}}\right), 7.53\left(\mathrm{~s}, 2 \mathrm{H}_{\mathrm{d}}\right), 6.55\left(\mathrm{~s}, 4 \mathrm{H}_{\mathrm{e}}\right)$. 


\section{S2. NMR Spectra}

Proton nuclear magnetic resonance $\left({ }^{1} \mathrm{H}\right.$ NMR) spectra were recorded at room temperature on a Bruker ${ }^{\circledR}$ DRX400 spectrometer at $400 \mathrm{MHz}$ (instrument parameters: field strength: $9.2 \mathrm{~T}$; RF-Console: DRX 3-channel; magnet: shielded superconducting; probe: nature, $5.0 \mathrm{~mm} \mathrm{BBO-}{ }^{1} \mathrm{H} /{ }^{15} \mathrm{~N}-{ }^{31} \mathrm{P}$; type, double-resonance; temperature range, $\left.-100-180{ }^{\circ} \mathrm{C}\right) .{ }^{1} \mathrm{H}$ NMR data are reported as follows: chemical shift $(\delta, \mathrm{ppm})$, multiplicity $(\mathrm{s}=\mathrm{singlet}$, br $\mathrm{s}=$ broad singlet, $\mathrm{d}=$ doublet, $\mathrm{dd}=$ doublet of doublets, $\mathrm{ddd}=$ doublet of doublet of doublets, app dt $=$ apparent doublet of triplets, app $\mathrm{td}=$ apparent triplet of doublets, $\mathrm{m}=$ multiplet), coupling constant(s) $(\mathrm{J}$, Hz), and integration. Chemical shift values were calibrated relative to residual solvent resonances $\left(\mathrm{CHCl}_{3}: \delta_{\mathrm{H}}=7.26 \mathrm{ppm}\right.$; DMSO: $\delta_{\mathrm{H}}=2.50 \mathrm{ppm}$ ) as the internal standard. All NMR data were collected and plotted within the Bruker ${ }^{\circledR}$ TopSpin $^{\mathrm{TM}}$ v4.0.6 software suite.

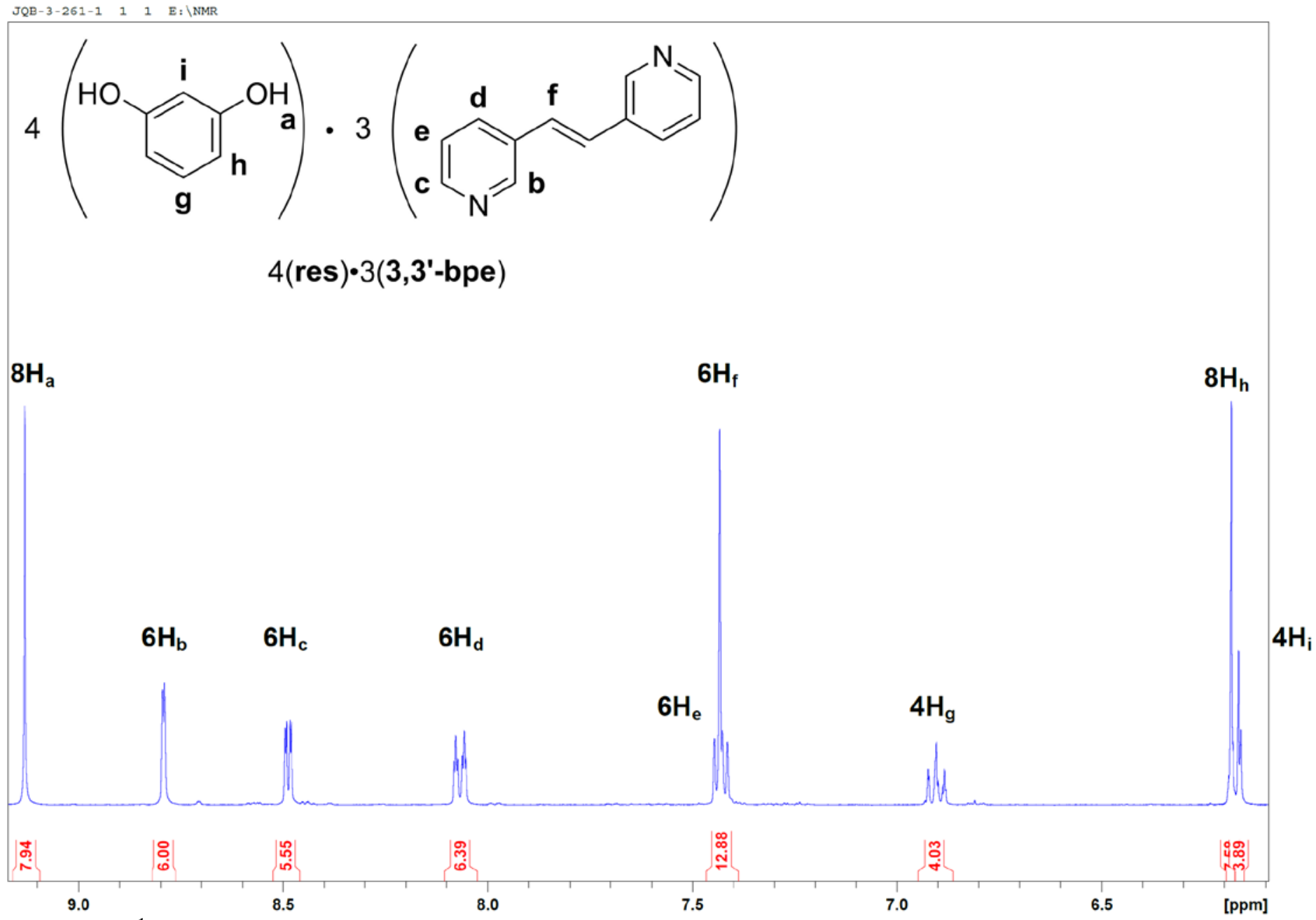

Figure S1. ${ }^{1} \mathrm{H}$ NMR spectrum of 4(res) $3(3,3$ '-bpe). The horizontal axis is calibrated relative to DMSO. 


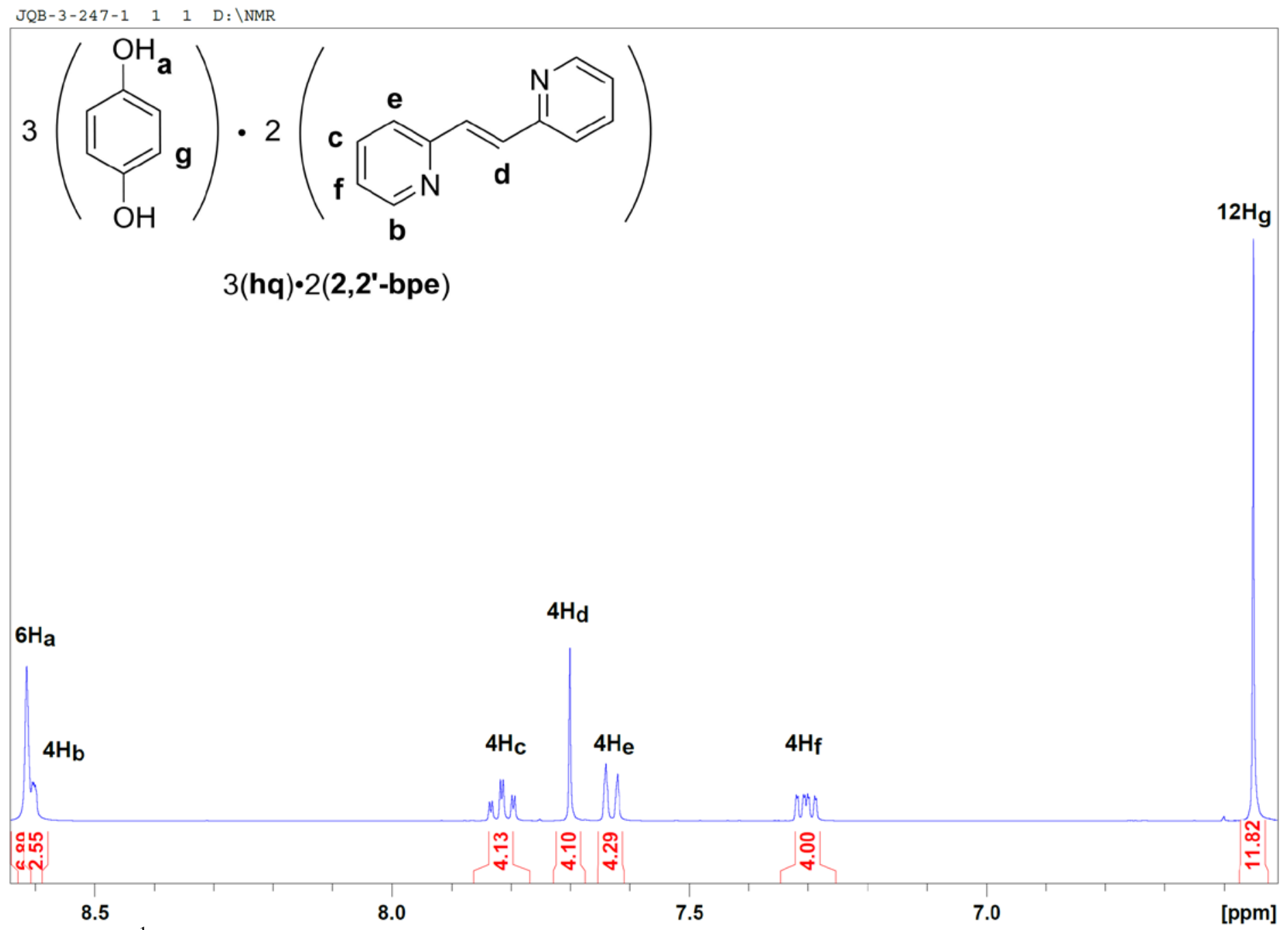

Figure S2. ${ }^{1} \mathrm{H}$ NMR (400 MHz, DMSO- $\left.d_{6}\right)$ spectrum of 3(hq)·2(2,2'-bpe). The horizontal axis is calibrated relative to DMSO. 


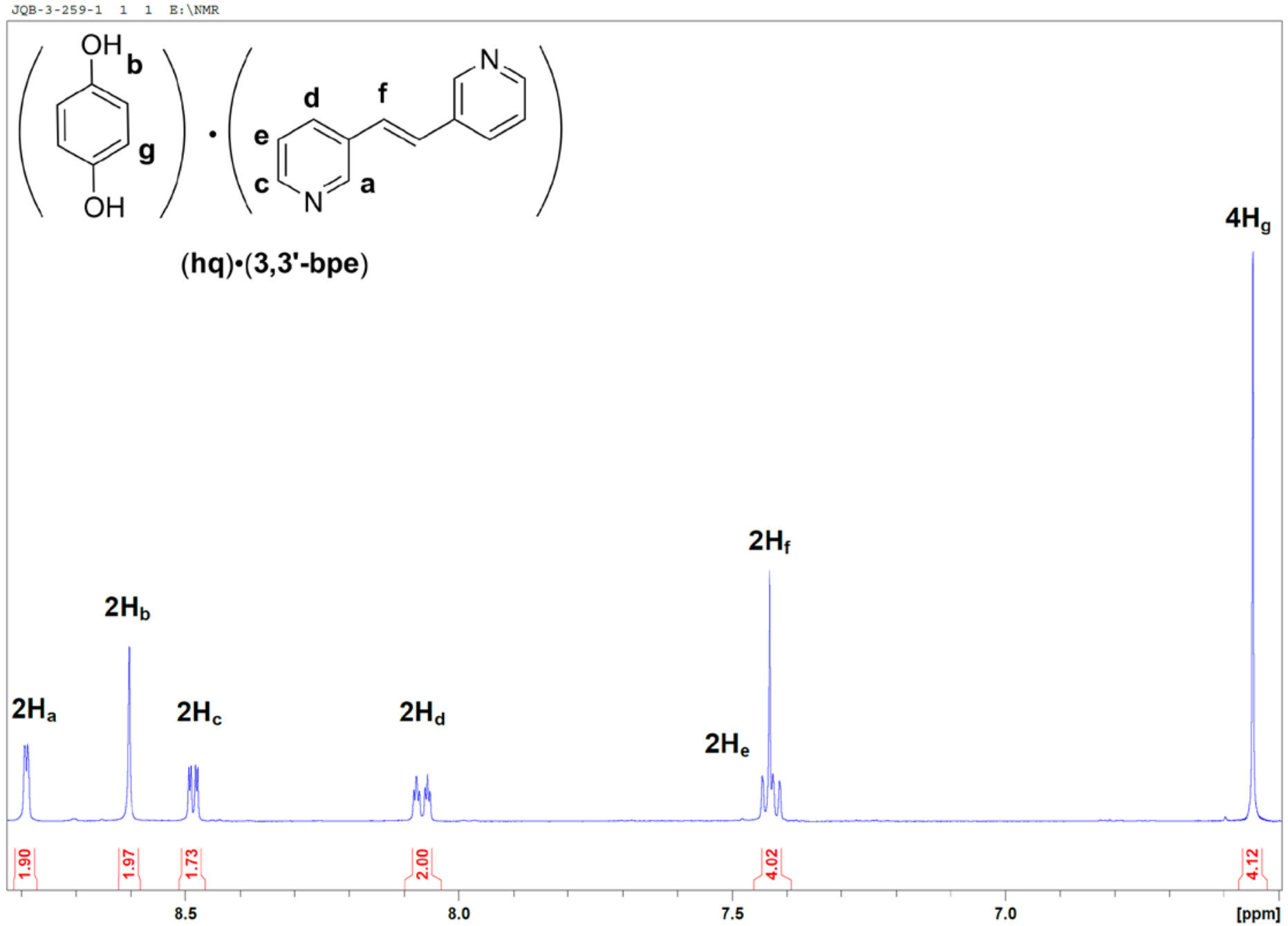

Figure S3. ${ }^{1} \mathrm{H}$ NMR spectrum of (hq) (3,3'-bpe). The horizontal axis is calibrated relative to DMSO. 


\section{S4. pXRD Data}

Powder X-ray diffraction data were collected at room temperature on a Bruker ${ }^{\circledR}$ D8 Advance X-ray diffractometer on samples mounted on glass slides. Each sample was finely ground using an agate mortar and pestle prior to mounting. Instrument parameters: radiation wavelength, $\mathrm{CuK} \alpha(\lambda=1.5418 \AA)$; scan type, coupled TwoTheta/Theta; scan mode, continuous PSD fast; scan range, 5-40 two-theta; step size, $0.02^{\circ}$; voltage, $40 \mathrm{kV}$; current, $30 \mathrm{~mA}$. Background subtractions were applied to all experimentally collected data within the Bruker ${ }^{\circledR}$ DIFFRAC.EVA v3.1 software suite. All data were plotted in Microsoft ${ }^{\circledR}$ Excel 2016. Simulated pXRD patterns were calculated from scXRD data within the CCDC Mercury ${ }^{2}$ software suite.

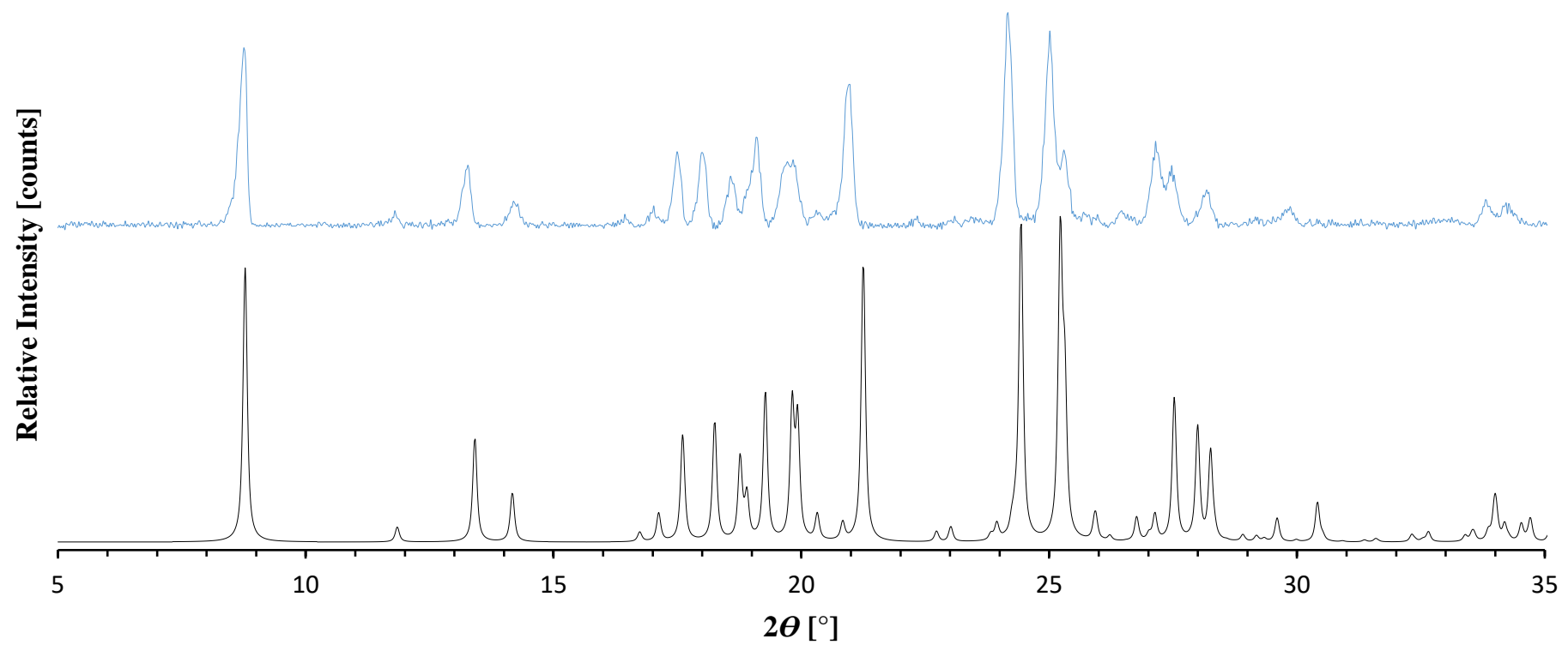

Figure S4. Experimental (top) and simulated (bottom) pXRD patterns for (res) (2,2'-bpe). The bottom pattern is simulated from CCDC 141324.

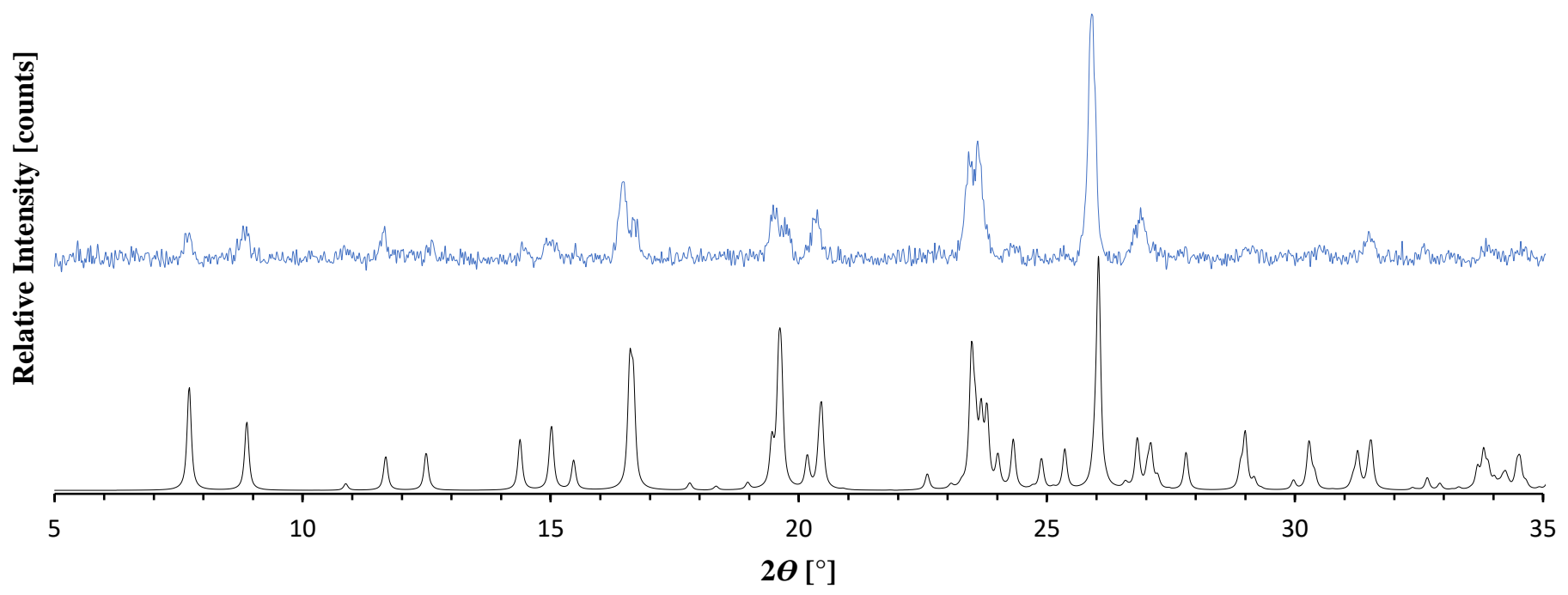

Figure S5. Experimental (top) and simulated (bottom) pXRD patterns for (1,3-di-I-tFb)·(3,3’-bpe). 


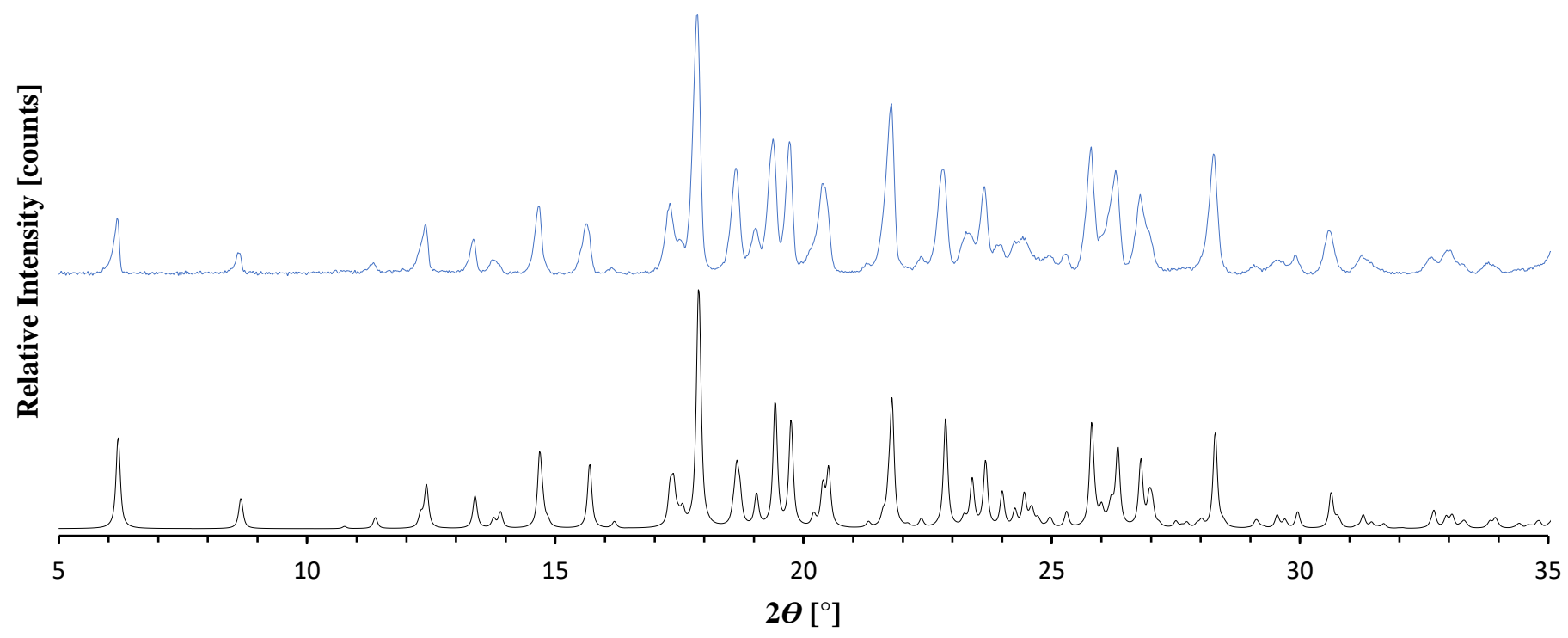

Figure S6. Experimental (top) and simulated (bottom) pXRD patterns for 4(res)·3(3,3’-bpe).

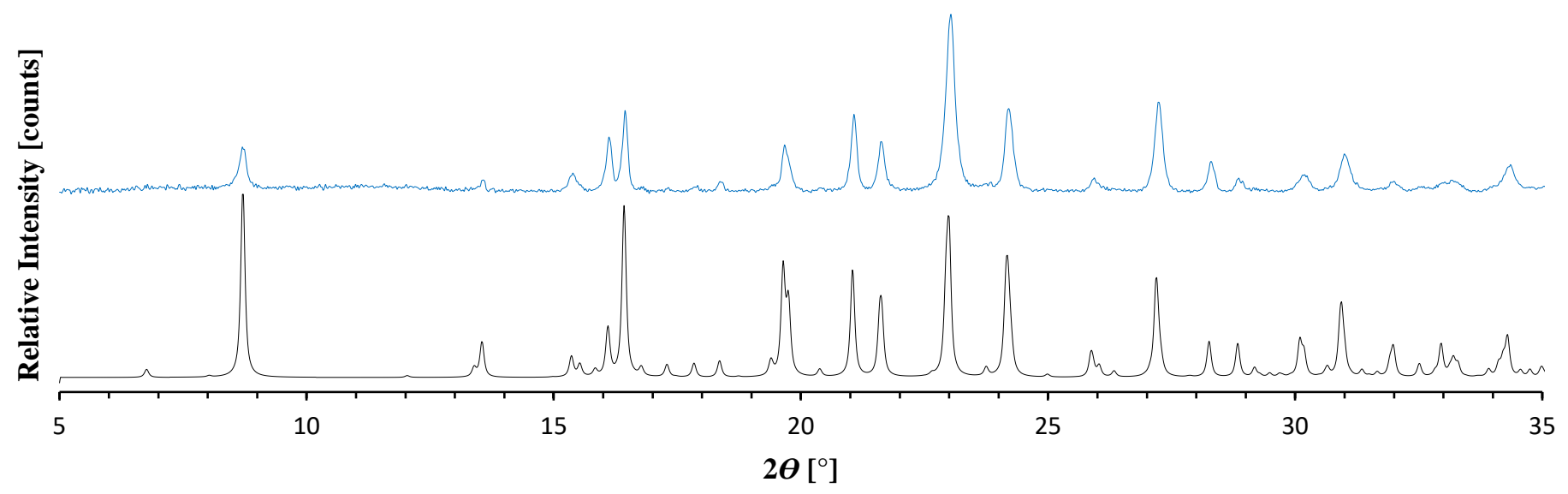

Figure S7. Experimental (top) and simulated (bottom) pXRD patterns for (1,3-di-I-tFb)·(4,4'-bpe). 


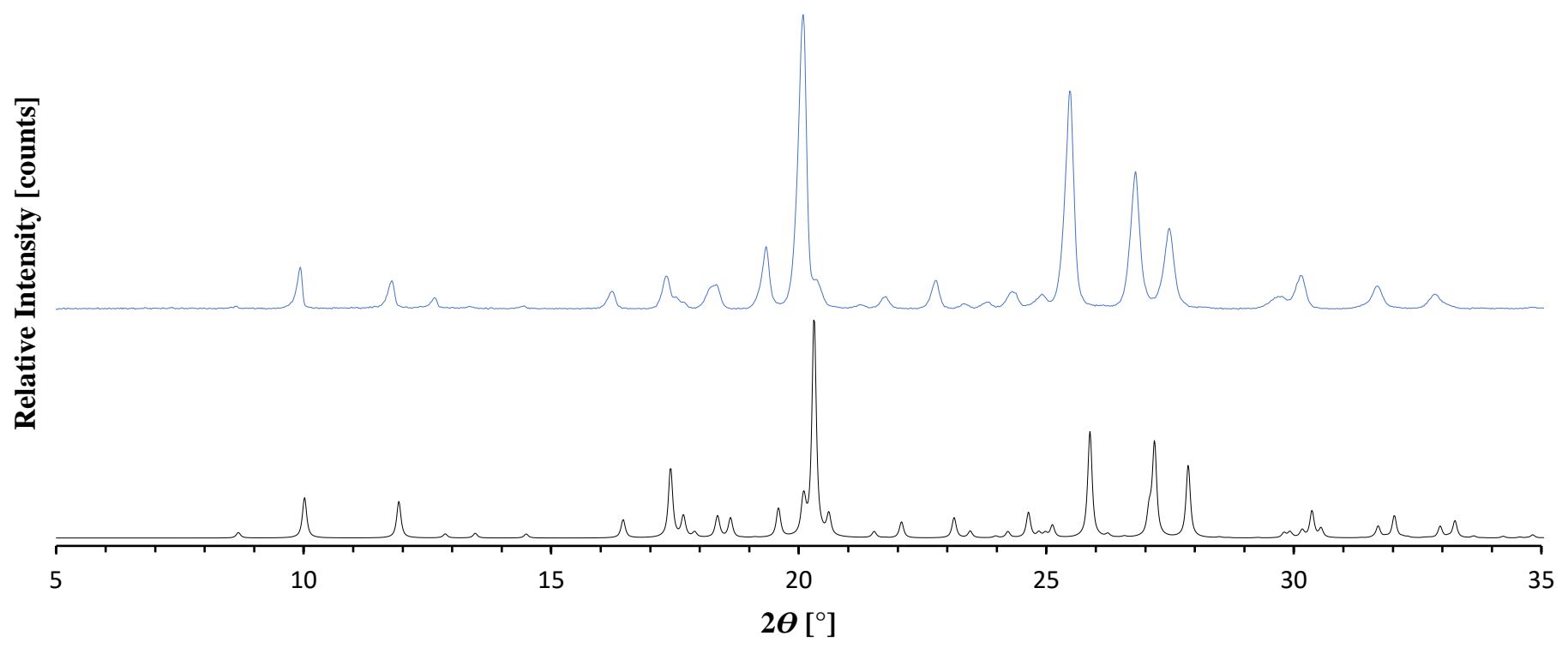

Figure S8. Experimental (top) and simulated (bottom) pXRD patterns for (res)·(4,4'-bpe). The bottom pattern is simulated from CCDC 141321.

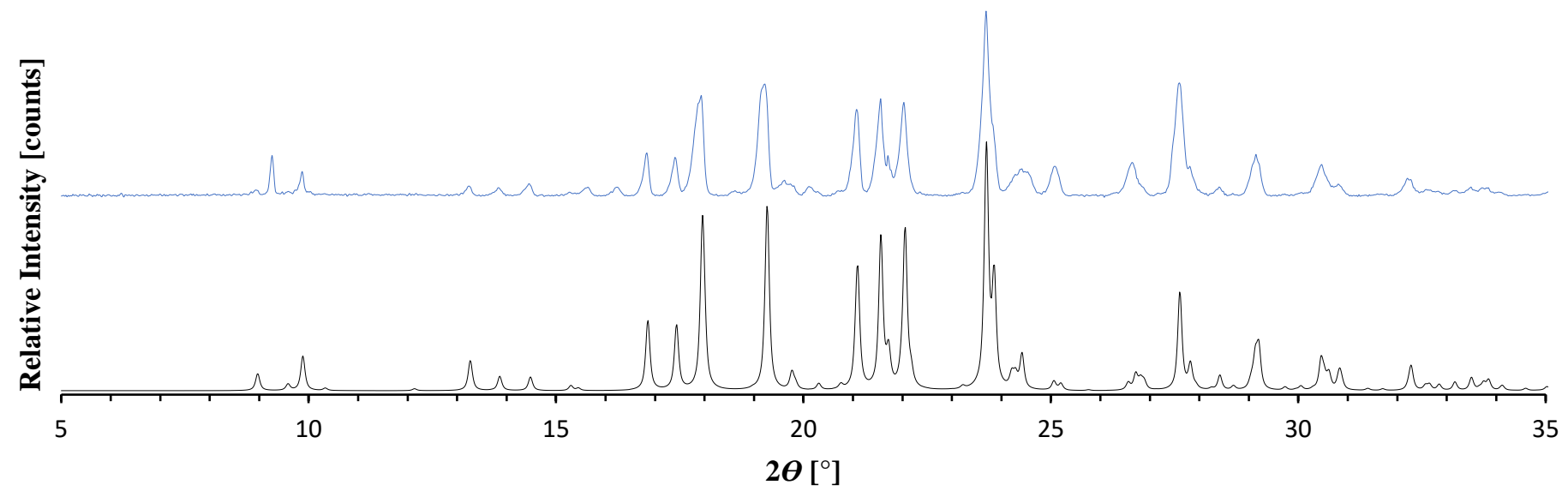

Figure S9. Experimental (top) and simulated (bottom) pXRD patterns for 3(hq)·2(2,2'-bpe).

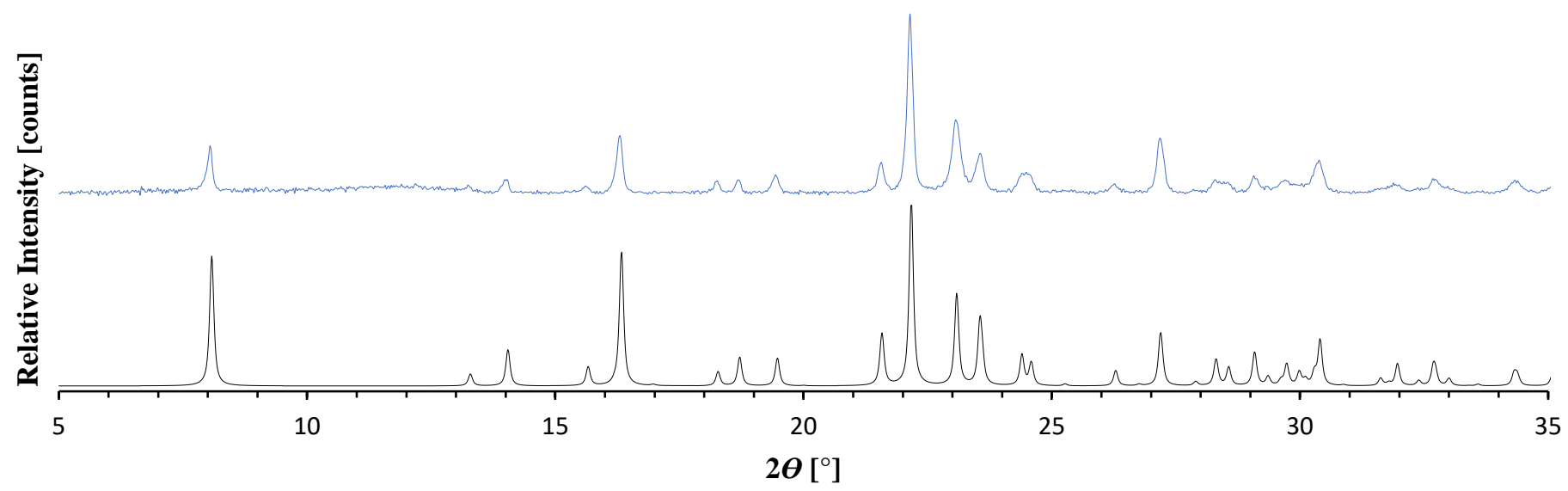

Figure S10. Experimental (top) and simulated (bottom) pXRD patterns for (1,4-di-I-tFb)·(3,3’-bpe). 


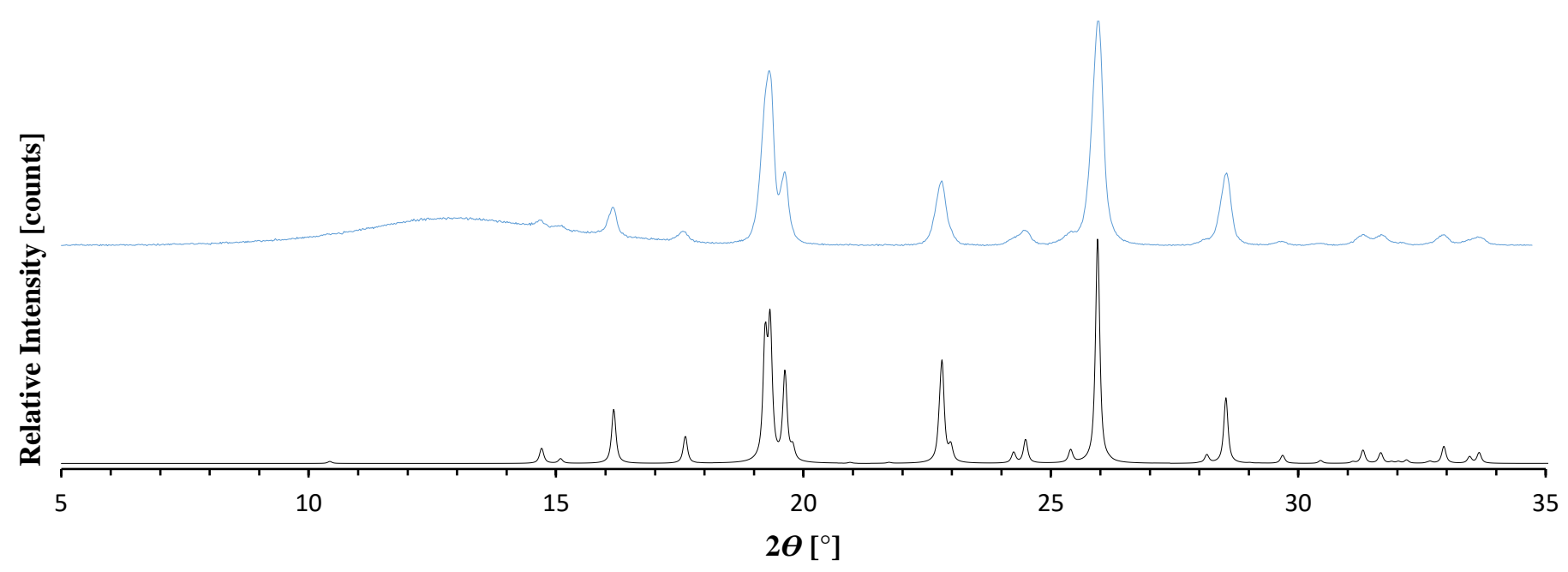

Figure S11. Experimental (top) and simulated (bottom) pXRD patterns for (hq)'(3,3'-bpe).

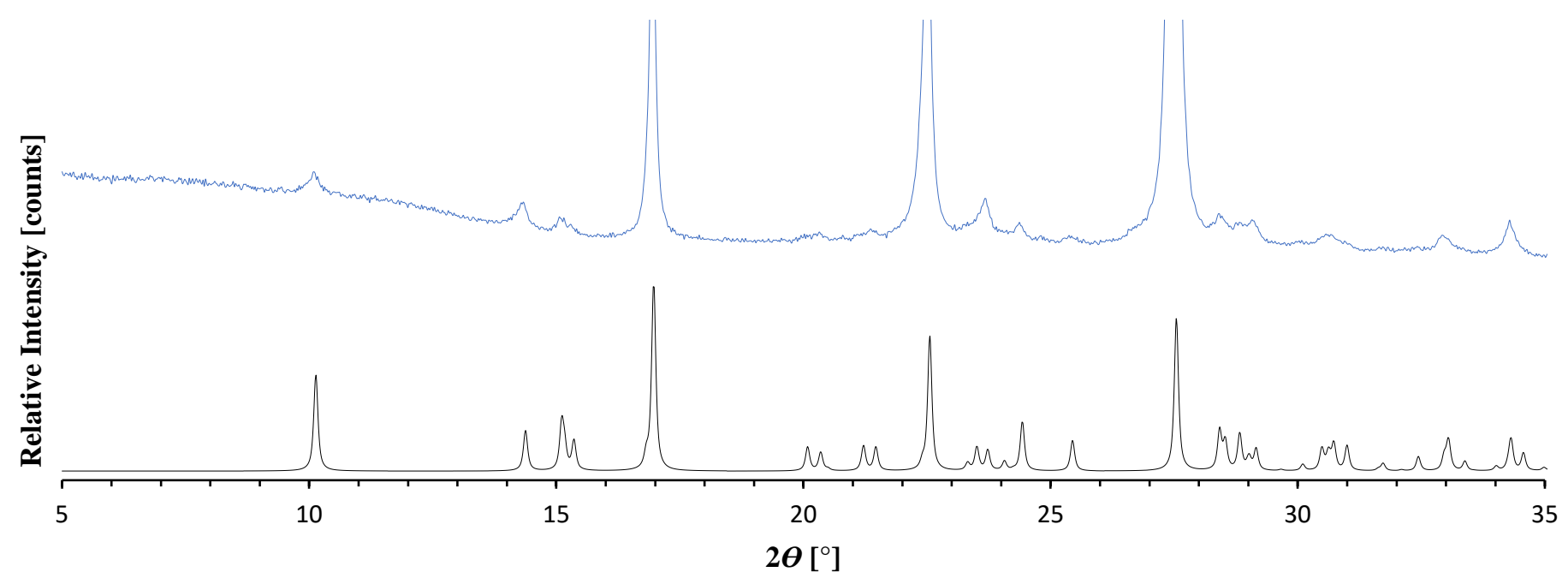

Figure S12. Experimental (top) and simulated (bottom) pXRD patterns for (1,4-di-I-tFb) (4,4'-bpe). The bottom pattern was simulated from CCDC 245122.

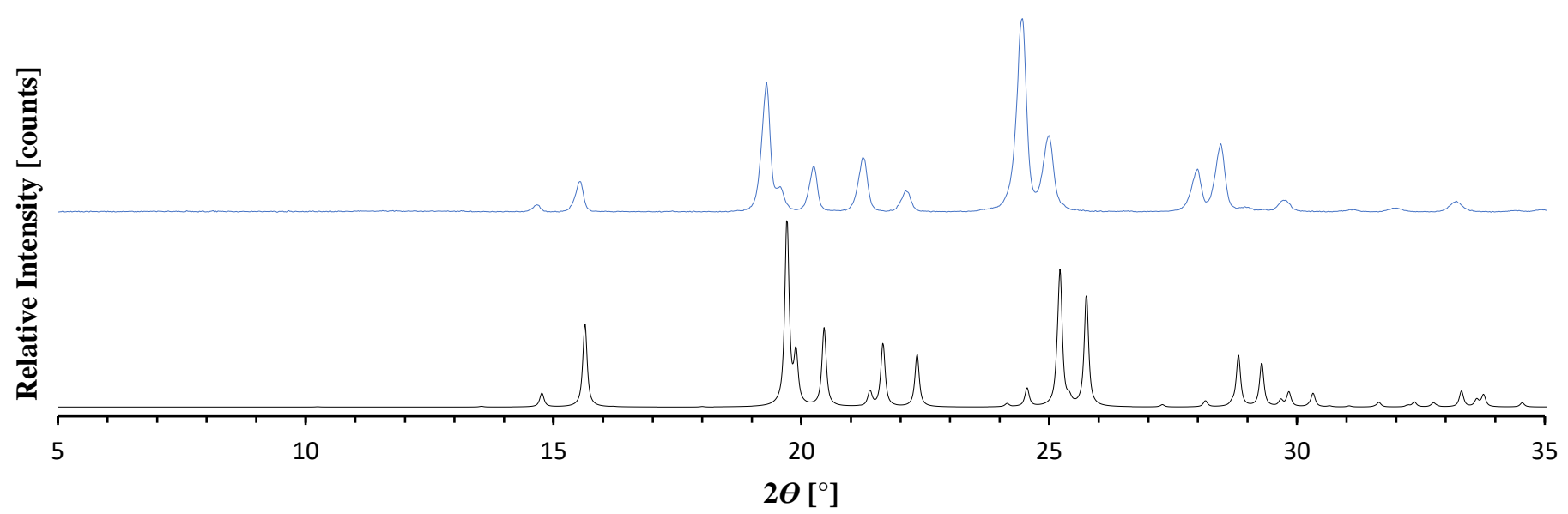

Figure S13. Experimental (top) and simulated (bottom) pXRD patterns for (hq) $\left(\mathbf{4 , 4} \mathbf{4}^{\prime}\right.$-bpe). The bottom pattern was simulated from CCDC 730431. 


\section{S4. scXRD Data}

Single-crystal X-ray diffraction data were collected on either a Bruker ${ }^{\circledR}$ Nonius-Kappa $^{\circledR}$ APEX II CCD or a Bruker $^{\circledR}$ Nonius-Kappa ${ }^{\circledR}$ CCD diffractometer, each equipped with an Oxford Cryosystems ${ }^{\circledR} 700$ series cold $\mathrm{N}_{2}$ gas stream cooling system. Data were collected at either room temperature (296.15 K) or low temperature (150.15 K) using graphite-monochromated MoK $\alpha$ radiation $(\lambda=0.71073 \AA)$. Crystals were mounted in Paratone ${ }^{\circledR}$ oil on a MiTeGen $^{\odot}$ magnetic mount. Data collection strategies for ensuring maximum data redundancy and completeness were calculated using the Bruker ${ }^{\circledR}$ Apex $\mathrm{II}^{\mathrm{TM}}$ software suite. Data collection, initial indexing, frame integration, Lorentz-polarization corrections and final cell parameter calculations were likewise accomplished using the Apex II software suite. Multi-scan absorption corrections were performed using SADABS. ${ }^{3}$ Structure solution and refinement were accomplished using SHELXT ${ }^{4}$ and SHELXL, ${ }^{5}$ respectively, within the Olex ${ }^{6}$ graphical user interface. Space groups were unambiguously verified using the PLATON ${ }^{\circledR 7}$ executable. All nonhydrogen atoms were refined anisotropically. All hydrogen atoms were attached via a riding model at calculated positions using suitable HFIX commands. The occupancies of the major and minor positions for the disordered $\mathrm{C}=\mathrm{C}$ core within $(\mathbf{1}, \mathbf{3}-\mathbf{d i}-\mathbf{I}-\mathbf{t F b}) \cdot\left(\mathbf{4 , 4}\right.$ '-bpe) and $3(\mathbf{h q}) \cdot \mathbf{2}\left(\mathbf{2}, \mathbf{2}^{\prime}\right.$-bpe $)$ converged to their respective ratios after each was identified in the difference map and freely refined. Figures of all structures were rendered in the CCDC Mercury $^{2}$ software suite.

Table S1. Crystal data and structure refinement statistics for (1,3-di-I-tFb) (3,3'-bpe).

\begin{tabular}{|c|c|}
\hline CCDC deposition number & 1985176 \\
\hline Empirical formula & $\mathrm{C}_{18} \mathrm{H}_{10} \mathrm{~F}_{4} \mathrm{I}_{2} \mathrm{~N}_{2}$ \\
\hline Formula weight $/ \mathrm{g} \cdot \mathrm{mol}^{-1}$ & 584.08 \\
\hline Temperature/K & 296.15 \\
\hline Crystal system & triclinic \\
\hline Space group & $P-1$ \\
\hline$a / \AA$ & $7.9685(8)$ \\
\hline$b / \AA$ & $10.4506(10)$ \\
\hline$c / \AA ̊$ & $11.6746(12)$ \\
\hline$\alpha /^{\circ}$ & $96.155(5)$ \\
\hline$\beta /^{\circ}$ & $97.299(5)$ \\
\hline$\gamma /{ }^{\circ}$ & $105.554(5)$ \\
\hline Volume $/ \AA^{3}$ & $918.80(16)$ \\
\hline $\mathrm{Z}$ & 2 \\
\hline$\rho_{\text {calc }} / \mathrm{g} \cdot \mathrm{cm}^{-3}$ & 2.111 \\
\hline$\mu / \mathrm{mm}^{-1}$ & 3.465 \\
\hline$F(000)$ & 548 \\
\hline Crystal size $/ \mathrm{mm}^{3}$ & $0.18 \times 0.15 \times 0.12$ \\
\hline Radiation & $\operatorname{MoK} \alpha(\lambda=0.71073)$ \\
\hline $2 \Theta$ range for data collection/ ${ }^{\circ}$ & 5.752 to 50.994 \\
\hline Index ranges & $-9 \leq \mathrm{h} \leq 9,-12 \leq \mathrm{k} \leq 12,-11 \leq 1 \leq 14$ \\
\hline Reflections collected & 5349 \\
\hline Independent reflections & $3407\left[R_{\text {int }}=0.0174, R_{\text {sigma }}=0.0322\right]$ \\
\hline Data/restraints/parameters & $3407 / 0 / 235$ \\
\hline Goodness-of-fit on $F^{2}$ & 1.054 \\
\hline \multirow[t]{2}{*}{ Final $R$ indices $[I \geq 2 \sigma(I)]$} & $R_{1}=0.0283$ \\
\hline & $w R_{2}=0.0610$ \\
\hline \multirow[t]{2}{*}{$R$ indices (all data) } & $R_{1}=0.0456$ \\
\hline & $w R_{2}=0.0658$ \\
\hline Largest diff. peak/hole/e $\cdot \AA^{-3}$ & $0.52 /-0.81$ \\
\hline
\end{tabular}


Table S2. Crystal data and structure refinement statistics for 4(res) $3(3,3$ '-bpe).

\begin{tabular}{|c|c|}
\hline CCDC deposition number & 1985202 \\
\hline Empirical formula & $\mathrm{C}_{30} \mathrm{H}_{27} \mathrm{~N}_{3} \mathrm{O}_{4}$ \\
\hline Formula weight $/ \mathrm{g} \cdot \mathrm{mol}^{-1}$ & 493.54 \\
\hline Temperature/K & 296.15 \\
\hline Crystal system & triclinic \\
\hline Space group & $P-1$ \\
\hline$a / \AA$ & $8.3960(8)$ \\
\hline$b / \AA$ & $10.9807(11)$ \\
\hline$c / \AA$ & $15.4072(15)$ \\
\hline$\alpha /^{\circ}$ & $109.631(5)$ \\
\hline$\beta /^{\circ}$ & $98.380(5)$ \\
\hline$\gamma /{ }^{\circ}$ & $94.853(5)$ \\
\hline Volume $/ \AA^{3}$ & $1309.9(2)$ \\
\hline $\mathrm{Z}$ & 2 \\
\hline$\rho_{\text {calc }} / \mathrm{g} \cdot \mathrm{cm}^{-3}$ & 1.251 \\
\hline$\mu / \mathrm{mm}^{-1}$ & 0.084 \\
\hline$F(000)$ & 520 \\
\hline Crystal size $/ \mathrm{mm}^{3}$ & $0.36 \times 0.25 \times 0.06$ \\
\hline Radiation & $\operatorname{MoK} \alpha(\lambda=0.71073)$ \\
\hline $2 \Theta$ range for data collection ${ }^{\circ}$ & 3.98 to 49.998 \\
\hline Index ranges & $-9 \leq \mathrm{h} \leq 9,-12 \leq \mathrm{k} \leq 13,-15 \leq 1 \leq 18$ \\
\hline Reflections collected & 7275 \\
\hline Independent reflections & $4582\left[R_{\mathrm{int}}=0.0205, R_{\mathrm{sigma}}=0.0355\right]$ \\
\hline Data/restraints/parameters & $4582 / 0 / 338$ \\
\hline Goodness-of-fit on $F^{2}$ & 1.059 \\
\hline Final $R$ indices $[I \geq 2 \sigma(I)]$ & $R_{1}=0.0436$ \\
\hline & $w R_{2}=0.1023$ \\
\hline$R$ indices (all data) & $\begin{array}{l}R_{1}=0.0669 \\
w R_{2}=0.1113\end{array}$ \\
\hline Largest diff. peak/hole/e $\cdot \AA^{-3}$ & $0.20 /-0.16$ \\
\hline
\end{tabular}


Table S3. Crystal data and structure refinement statistics for (1,3-di-I-tFb) (4,4'-bpe).

\begin{tabular}{|c|c|}
\hline $\begin{array}{l}\text { CCDC deposition number } \\
\text { Empirical formula }\end{array}$ & $\begin{array}{l}1985182 \\
\mathrm{C}_{18} \mathrm{H}_{10} \mathrm{~F}_{4} \mathrm{I}_{2} \mathrm{~N}_{2}\end{array}$ \\
\hline Formula weight $/ \mathrm{g} \cdot \mathrm{mol}^{-1}$ & 584.08 \\
\hline Temperature/K & 296.15 \\
\hline Crystal system & monoclinic \\
\hline Space group & $P 22_{1} / n$ \\
\hline$a / \AA$ & $14.9362(15)$ \\
\hline$b / \AA$ & $6.1869(6)$ \\
\hline$c / \AA$ & $20.619(2)$ \\
\hline$\alpha /^{\circ}$ & 90 \\
\hline$\beta /^{\circ}$ & $100.286(5)$ \\
\hline$\gamma /{ }^{\circ}$ & 90 \\
\hline Volume $/ \AA^{3}$ & 1874.8(3) \\
\hline $\mathrm{Z}$ & 4 \\
\hline$\rho_{\text {calc }} / \mathrm{g} \cdot \mathrm{cm}^{-3}$ & 2.069 \\
\hline$\mu / \mathrm{mm}^{-1}$ & 3.396 \\
\hline$F(000)$ & 1096 \\
\hline Crystal size $/ \mathrm{mm}^{3}$ & $0.32 \times 0.18 \times 0.08$ \\
\hline Radiation & $\operatorname{MoK} \alpha(\lambda=0.71073)$ \\
\hline $2 \Theta$ range for data collection $/{ }^{\circ}$ & 5.544 to 50 \\
\hline Index ranges & $-17 \leq \mathrm{h} \leq 12,-7 \leq \mathrm{k} \leq 7,-23 \leq 1 \leq 24$ \\
\hline Reflections collected & 9025 \\
\hline Independent reflections & $3290\left[R_{\text {int }}=0.0274, R_{\text {sigma }}=0.0264\right]$ \\
\hline Data/restraints/parameters & $3290 / 0 / 239$ \\
\hline Goodness-of-fit on $F^{2}$ & 1.067 \\
\hline Final $R$ indices $[I \geq 2 \sigma(I)]$ & $R_{1}=0.0254$ \\
\hline & $w R_{2}=0.0618$ \\
\hline$R$ indices (all data) & $\begin{array}{l}R_{1}=0.0325 \\
w R_{2}=0.0649\end{array}$ \\
\hline Largest diff. peak/hole/e $\cdot \AA^{-3}$ & $0.40 /-0.75$ \\
\hline
\end{tabular}


Table S4. Crystal data and structure refinement statistics for 3(hq) -2(2,2'-bpe).

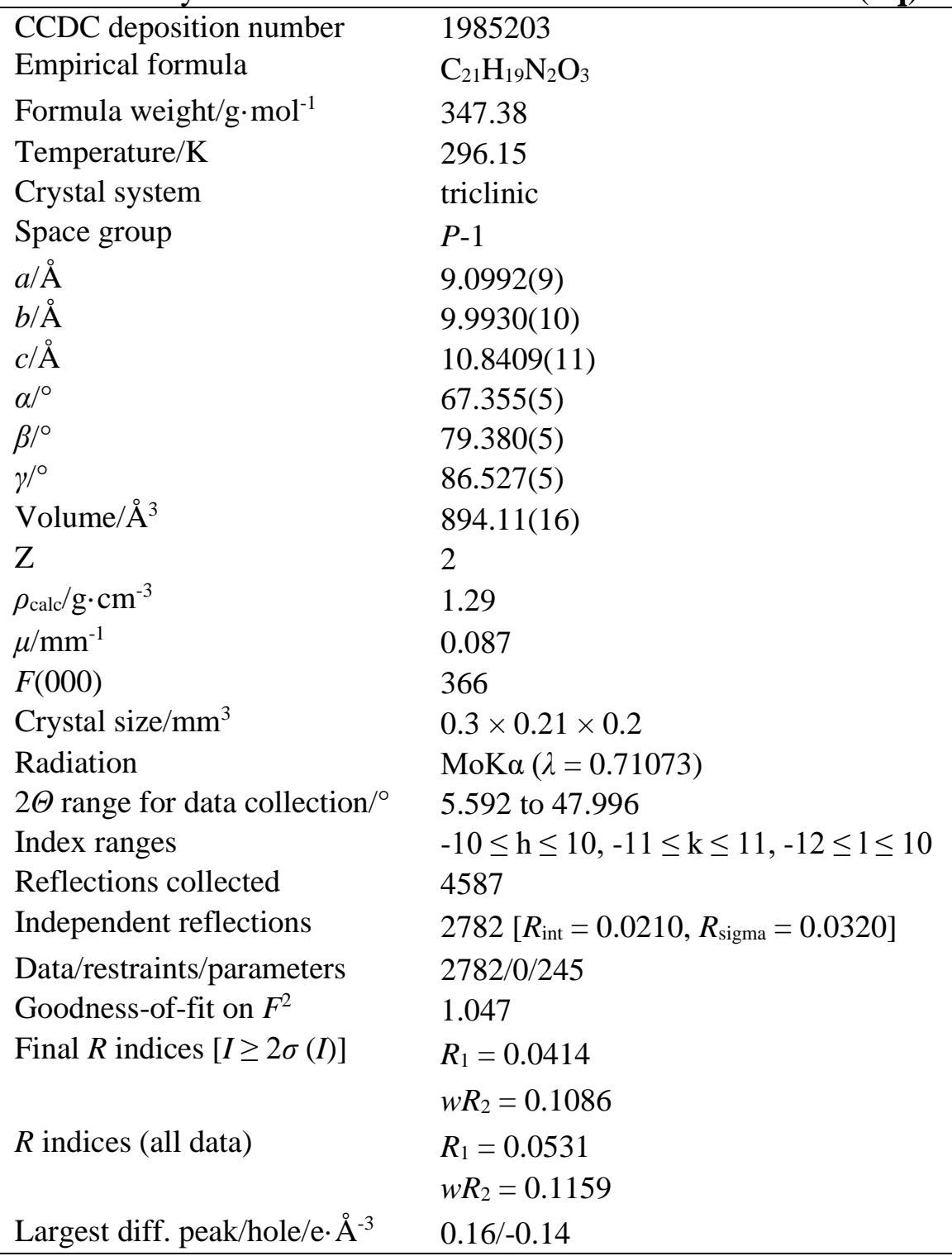


Table S5. Crystal data and structure refinement statistics for (1,4-di-I-tFb) (3,3'-bpe).

\begin{tabular}{|c|c|}
\hline CCDC deposition number & 1985188 \\
\hline Empirical formula & $\mathrm{C}_{18} \mathrm{H}_{10} \mathrm{~F}_{4} \mathrm{I}_{2} \mathrm{~N}_{2}$ \\
\hline Formula weight $/ \mathrm{g} \cdot \mathrm{mol}^{-1}$ & 584.08 \\
\hline Temperature/K & 296.15 \\
\hline Crystal system & monoclinic \\
\hline Space group & $P 2_{1} / c$ \\
\hline$a / \AA$ & $11.1810(11)$ \\
\hline$b / \AA$ & $6.2446(6)$ \\
\hline$c / \AA$ & $13.6142(14)$ \\
\hline$\alpha /^{\circ}$ & 90 \\
\hline$\beta /^{\circ}$ & $102.044(5)$ \\
\hline$\gamma /{ }^{\circ}$ & 90 \\
\hline Volume $/ \AA^{3}$ & 929.63(16) \\
\hline $\mathrm{Z}$ & 2 \\
\hline$\rho_{\text {call }} / \mathrm{g} \cdot \mathrm{cm}^{-3}$ & 2.087 \\
\hline$\mu / \mathrm{mm}^{-1}$ & 3.425 \\
\hline$F(000)$ & 548 \\
\hline Crystal size/mm $\mathrm{mm}^{3}$ & $0.26 \times 0.18 \times 0.1$ \\
\hline Radiation & $\operatorname{MoK} \alpha(\lambda=0.71073)$ \\
\hline $2 \Theta$ range for data collection/ ${ }^{\circ}$ & 6.12 to 50 \\
\hline Index ranges & $-13 \leq \mathrm{h} \leq 13,-7 \leq \mathrm{k} \leq 7,-12 \leq 1 \leq 16$ \\
\hline Reflections collected & 4724 \\
\hline Independent reflections & $1628\left[R_{\mathrm{int}}=0.0236, R_{\mathrm{sigma}}=0.0223\right]$ \\
\hline Data/restraints/parameters & $1628 / 0 / 118$ \\
\hline Goodness-of-fit on $F^{2}$ & 1.06 \\
\hline Final $R$ indices $[I \geq 2 \sigma(I)]$ & $R_{1}=0.0219$ \\
\hline & $w R_{2}=0.0532$ \\
\hline$R$ indices (all data) & $\begin{array}{l}R_{1}=0.0268 \\
w R_{2}=0.0553\end{array}$ \\
\hline Largest diff. peak/hole/e $\cdot \AA^{-3}$ & $0.34 /-0.52$ \\
\hline
\end{tabular}


Table S6. Crystal data and structure refinement statistics for (hq) (3,3'-bpe).

\begin{tabular}{|c|c|}
\hline CCDC deposition number & 1985199 \\
\hline Empirical formula & $\mathrm{C}_{18} \mathrm{H}_{16} \mathrm{~N}_{2} \mathrm{O}_{2}$ \\
\hline Formula weight $/ \mathrm{g} \cdot \mathrm{mol}^{-1}$ & 292.33 \\
\hline Temperature/K & 150.15 \\
\hline Crystal system & triclinic \\
\hline Space group & $P-1$ \\
\hline$a / \AA$ & $6.1637(6)$ \\
\hline$b / \AA$ & $7.1041(7)$ \\
\hline$c / \AA$ & $8.7870(9)$ \\
\hline$\alpha /^{\circ}$ & $80.619(5)$ \\
\hline$\beta /^{\circ}$ & $77.571(5)$ \\
\hline$\gamma /{ }^{\circ}$ & $86.851(5)$ \\
\hline Volume $/ \AA^{3}$ & $370.64(6)$ \\
\hline $\mathrm{Z}$ & 1 \\
\hline$\rho_{\text {calc }} / \mathrm{g} \cdot \mathrm{cm}^{-3}$ & 1.31 \\
\hline$\mu / \mathrm{mm}^{-1}$ & 0.087 \\
\hline$F(000)$ & 154 \\
\hline Crystal size/mm $\mathrm{mm}^{3}$ & $0.155 \times 0.1 \times 0.09$ \\
\hline Radiation & $\operatorname{MoK} \alpha(\lambda=0.71073)$ \\
\hline $2 \Theta$ range for data collection/ ${ }^{\circ}$ & 4.806 to 55.764 \\
\hline Index ranges & $-8 \leq \mathrm{h} \leq 8,-9 \leq \mathrm{k} \leq 9,-9 \leq 1 \leq 11$ \\
\hline Reflections collected & 5110 \\
\hline Independent reflections & $1708\left[R_{\text {int }}=0.0226, R_{\text {sigma }}=0.0245\right]$ \\
\hline Data/restraints/parameters & $1708 / 0 / 101$ \\
\hline Goodness-of-fit on $F^{2}$ & 1.035 \\
\hline Final $R$ indices $[I \geq 2 \sigma(I)]$ & $R_{1}=0.0424$ \\
\hline & $w R_{2}=0.1092$ \\
\hline$R$ indices (all data) & $\begin{array}{l}R_{1}=0.0490 \\
w R_{2}=0.1136\end{array}$ \\
\hline Largest diff. peak/hole/e $\cdot \AA^{-3}$ & $0.18 /-0.17$ \\
\hline
\end{tabular}


S4. Bond Metrics and Melting Point Tables.

Table S7. Hydrogen bond metrics and photoreactivity results for H-bonded cocrystals.

\begin{tabular}{|c|c|c|c|c|c|}
\hline Cocrystal $^{[\mathrm{a}]}$ & H-Bond & $d(\mathrm{O} \cdots \mathrm{A}) / \AA^{[\mathrm{b}]}$ & $\boldsymbol{\Theta}(\mathbf{O}-\mathbf{H} \cdots \mathbf{A}) /^{\mathrm{o}}{ }^{[\mathrm{b}]}$ & prs $^{*[c]}$ & Photoreactivity \\
\hline \multirow[t]{4}{*}{ 2(cat)·(2,2'-bpe) (1025307) } & O1-H1A $\cdots$ N1 & $2.658(2)$ & 167.2 & 13 & active \\
\hline & O3-H3A $\cdots$ N2 & $2.624(2)$ & 172.4 & 15 & \\
\hline & $\mathrm{O} 2-\mathrm{H} 2 \mathrm{~A} \cdots \mathrm{O} 3$ & $2.732(2)$ & 165.3 & 10 & \\
\hline & $\mathrm{O} 4-\mathrm{H} 4 \mathrm{~A} \cdots \mathrm{O} 1$ & $2.701(2)$ & 157.5 & 11 & \\
\hline \multirow[t]{2}{*}{ (cat)·(3,3’-bpe) (1860795) } & $\mathrm{O} 1-\mathrm{H} 1 \cdots \mathrm{N} 1$ & $2.751(2)$ & 169.3 & 10 & active \\
\hline & $\mathrm{O} 2-\mathrm{H} 2 \cdots \mathrm{N} 2$ & $2.745(2)$ & 158.7 & 11 & \\
\hline \multirow[t]{4}{*}{ (cat) $\cdot(4,4$ '-bpe) (244922) } & O1-H1O $\cdots \mathrm{N} 1$ & $2.733(2)$ & $170.0(1)$ & 11 & inert \\
\hline & $\mathrm{O} 2-\mathrm{H} 2 \mathrm{O} \cdots \mathrm{N} 4$ & $2.756(2)$ & 174.37(9) & 10 & \\
\hline & O3-H3O $\cdots$ N3 & $2.710(2)$ & $167.00(9)$ & 12 & \\
\hline & $\mathrm{O} 4-\mathrm{H} 4 \mathrm{O} \cdots \mathrm{N} 2$ & $2.749(2)$ & 172.67(9) & 10 & \\
\hline \multirow[t]{2}{*}{$($ res $) \cdot(2,2$ '-bpe $)(141324)$} & $\mathrm{O} 1-\mathrm{H} 01 \cdots \mathrm{N} 1$ & 2.806 & 160.8 & 9 & active \\
\hline & $\mathrm{O} 2-\mathrm{H} 02 \cdots \mathrm{N} 2$ & 2.779 & 166.88 & 9 & \\
\hline \multirow[t]{4}{*}{$4($ res $) \cdot 3(3,3 '$-bpe $)$} & $\mathrm{O} 1-\mathrm{H} 1 \cdots \mathrm{N} 1$ & $2.659(2)$ & 170.83 & 13 & inert \\
\hline & $\mathrm{O} 2-\mathrm{H} 2 \cdots \mathrm{N} 2$ & $2.822(2)$ & 170.44 & 8 & \\
\hline & O3-H3 $\cdots$ N3 & $2.737(2)$ & 174.0 & 11 & \\
\hline & $\mathrm{O} 4-\mathrm{H} 4 \cdots \mathrm{O} 1$ & $2.722(2)$ & 169.7 & 10 & \\
\hline \multirow[t]{2}{*}{ (res) $(\mathbf{4 , 4}$ '-bpe) (141321) } & $\mathrm{O} 1-\mathrm{H} 01 \cdots \mathrm{N} 1$ & $2.734(2)$ & 176.8 & 11 & active \\
\hline & $\mathrm{O} 2-\mathrm{H} 02 \cdots \mathrm{N} 2$ & $2.753(2)$ & 176.2 & 10 & \\
\hline \multirow[t]{3}{*}{$3(\mathbf{h q}) \cdot 2\left(2, \mathbf{2}^{\prime}-\mathbf{b p e}\right)$} & $\mathrm{O} 1-\mathrm{H} 1 \cdots \mathrm{N} 1$ & $2.730(2)$ & 170.5 & 11 & inert \\
\hline & $\mathrm{O} 2-\mathrm{H} 2 \mathrm{~A} \cdots \mathrm{N} 2$ & $2.808(2)$ & 164.9 & 9 & \\
\hline & O3-Н3А $\cdots$ O1 & $2.783(2)$ & 163.1 & 8 & \\
\hline (hq) $\cdot\left(3,3^{\prime}-\right.$-bpe $)$ & $\mathrm{O} 1-\mathrm{H} 1 \cdots \mathrm{N} 1$ & $2.744(2)$ & 164.4 & 11 & inert \\
\hline$(\mathbf{h q}) \cdot(\mathbf{4 , 4}$-bpe $)(730321)$ & $\mathrm{O} 11-\mathrm{H} 11 \mathrm{O} \cdots \mathrm{N} 1$ & $2.705(2)$ & 167.7 & 12 & inert \\
\hline
\end{tabular}

[a] CCDC deposition numbers for previously reported cocrystals are given in parentheses following the cocrystal formula; [b] A is the H-bond acceptor; [c]; prs $\equiv$ percent relative shortening $=\left\{1-d(\mathrm{~N} \cdots \mathrm{O}) /\left[r_{\mathrm{vdW}}(\mathrm{O})+\right.\right.$ $\left.\left.r_{\mathrm{vdW}}(\mathrm{N})\right]\right\} \cdot 100$, with $r_{\mathrm{vdW}}(\mathrm{N})=1.55 \AA$ and $r_{\mathrm{vdW}}(\mathrm{O})=1.52 \AA$. 
Table S8. Bond metrics and photoreactivity results for X-bonded cocrystals.

\begin{tabular}{|c|c|c|c|c|c|c|}
\hline Cocrystal $^{[\mathrm{a}]}$ & X-Bond & $d(\mathrm{~N} \cdots \mathrm{I}) / \AA$ & $\Theta(\mathrm{C}-\mathrm{I} \cdot \cdots \mathrm{N}) /^{\circ}$ & X-Bond Type & $\operatorname{prs}^{[\mathrm{b}]}$ & Photoreactivity \\
\hline 2(1,2-di-I-tFb)·(2,2'-bpe) (1860151) & $\mathrm{I} 1 \cdots \mathrm{N} 1$ & $3.040(3)$ & 173.9 & I & 14 & inert \\
\hline \multirow[t]{2}{*}{ 2(1,2-di-I-tFb)·(3,3’-bpe) (1864394) } & $\mathrm{I} 1 \cdots \mathrm{N} 1$ & $2.875(5)$ & 174.4 & I & 19 & inert \\
\hline & $\mathrm{I} 3 \cdots \mathrm{N} 2$ & $2.897(6)$ & 172.2 & I & 15 & \\
\hline \multirow[t]{2}{*}{$(\mathbf{1 , 2 - d i - I - t F b ) \cdot ( 4 , 4 ' - b p e ) ~ ( 1 8 6 3 4 2 8 ) ~}$} & $\mathrm{I} 1 \cdots \mathrm{N} 1$ & $2.807(2)$ & 177.2 & I & 20 & inert \\
\hline & $\mathrm{I} 2 \cdots \mathrm{N} 2$ & 2.886(3) & 177.4 & I & 18 & \\
\hline \multirow[t]{4}{*}{ 2(1,3-di-I-tFb)· 2(2,2'-bpe) (1940759) } & $\mathrm{I} 1 \cdots \mathrm{N} 1$ & $2.96(2)$ & 172.6 & I & 16 & active \\
\hline & $\mathrm{I} 2 \cdots \mathrm{N} 2$ & $3.00(1)$ & 174.2 & I & 15 & \\
\hline & I3 $\cdots$ N3 & $2.99(1)$ & 172.2 & I & 15 & \\
\hline & $\mathrm{I} 4 \cdots \mathrm{N} 4$ & $3.04(1)$ & 174.4 & I & 14 & \\
\hline \multirow[t]{2}{*}{$(1,3-d i-I-t F b) \cdot(3,3 ’-b p e)$} & $\mathrm{I} 1 \cdots \mathrm{N} 1$ & $2.942(4)$ & 168.6 & I & 17 & inert \\
\hline & $\mathrm{I} 2 \cdots \mathrm{N} 2$ & $3.009(3)$ & 165.4 & I & 15 & \\
\hline \multirow[t]{2}{*}{$(1,3-d i-I-t F b) \cdot(4,4 ’-b p e)$} & $\mathrm{I} 1 \cdots \mathrm{N} 1$ & 2.915(3) & 170.0 & I & 17 & inert \\
\hline & $\mathrm{I} 2 \cdots \mathrm{N} 2$ & $2.879(3)$ & 173.2 & I & 18 & \\
\hline \multirow[t]{2}{*}{$($ (1,4-di-I-tFb)·(2,2'-bpe) (1940762) } & $\mathrm{I} 1 \cdots \mathrm{N} 1$ & $2.967(8)$ & 177.1 & I & 16 & active \\
\hline & $\mathrm{I} 2 \cdots \mathrm{N} 2$ & 2.982(8) & 177.2 & I & 16 & \\
\hline$(1,4-d i-I-t F b) \cdot(3,3 '-b p e)$ & $\mathrm{I} 1 \cdots \mathrm{N} 1$ & 2.958(3) & 175.0 & I & 16 & inert \\
\hline$(\mathbf{1 , 4 - d i - I - t F b ) \cdot ( 4 , 4 ' - b p e )}(161324)$ & $\mathrm{I}(1) \cdots \mathrm{N}(1)$ & $2.810(5)$ & 179.3 & $\mathrm{I}$ & 20 & inert \\
\hline
\end{tabular}

[a] CCDC deposition numbers for previously reported cocrystals are given in parentheses following the cocrystal formula; [b] prs $\equiv$ percent relative shortening $=\left\{1-d(\mathrm{~N} \cdots \mathrm{I}) /\left[r_{\mathrm{vdW}}(\mathrm{I})+r_{\mathrm{vdW}}(\mathrm{N})\right]\right\} \cdot 100$, with $r_{\mathrm{vdW}}(\mathrm{N})=1.55 \AA$ and $r_{\mathrm{vdW}}(\mathrm{I})=1.98 \AA$.

Table S9. Melting point data for H-bonded cocrystals and coformers thereof. For previously reported compounds, the CCDC deposition numbers are given in parentheses following the structural formula.

\begin{tabular}{|c|c|c|c|}
\hline Compound & $\mathbf{m p} /{ }^{\circ} \mathrm{C}$ & Solvent & Reference \\
\hline cat $(185293)$ & $103-105$ & $\mathrm{PhH}$ & Bull. Chem. Soc. Jpn. 1964, 37, 564-568 \\
\hline \multirow[t]{3}{*}{ res $(168981)$} & $228-230$ & $\mathrm{EtOH}$ & Bulg. Chem. Commun. 2008, 40, 147-159 \\
\hline & $109-110$ & $\mathrm{PhH}$ & Tetrahedron 1958, 3, 316 \\
\hline & $128.0-128.5$ & $\mathrm{AcMe}$ & Helv. Chim. Acta 1957, 40, 758-778 \\
\hline \multirow[t]{2}{*}{ hq (1179128) } & $137.5-140.0$ & $\mathrm{PhH}$ & Aust. J. Chem. 1966, 19, 169-174 \\
\hline & $128-129$ & $\mathrm{Et}_{2} \mathrm{O}$ & Science of Synthesis 2006, 28, 367-506 \\
\hline 2,2'-bpe & $119-120$ & $\mathrm{PE}$ & J. Org. Chem. 1982, 47, 3497-3503 \\
\hline \multirow[t]{3}{*}{ 3,3'-bpe } & 90 & $\mathrm{PhH}$ & ChemPhysChem 2020, 21, 154-163 \\
\hline & 71 & $\mathrm{CH}_{2} \mathrm{Cl}_{2}$ & Heterocycles 1990, 7, 1271-1274 \\
\hline & $151-152$ & $\mathrm{PhH}$ & J. Am. Chem. Soc. 1948, 70, 2330-2333 \\
\hline \multirow[t]{3}{*}{ 4,4'-bpe } & $150-153$ & $\mathrm{CHCl}_{3}$ & Chem. Eur. J. 2003, 9, 3974-3983 \\
\hline & $149-150$ & $\mathrm{PhH}$ & Chem. Pharm. Bull. 1967, 15, 511-514 \\
\hline & 151-152 & $\mathrm{PhH}$ & J. Am. Chem. Soc. 1948, 70, 2330-2333 \\
\hline 2(cat)·(2,2'-bpe) (1025307) & $138-140$ & AcMe & ChemPhysChem 2020, 21, 154-163 \\
\hline (cat)·(3,3’-bpe) (1860795) & 124-125 (dec.) & AcMe & ChemPhysChem 2020, 21, 154-163 \\
\hline (cat) $\cdot(4,4$ '-bpe) $(244922)$ & 152-153 (dec.) & AcMe & ChemPhysChem 2020, 21, 154-163 \\
\hline (res) $\cdot\left(2,2^{\prime}\right.$-bpe) (141324) & $95-97$ & $\mathrm{MeOH}$ & This work \\
\hline $4($ res $) \cdot 3(3,3$ '-bpe $)$ & 122-130 (dec.) & $\mathrm{MeOH}$ & This work \\
\hline (res) $\cdot\left(4,4^{\prime}\right.$-bpe) $(141321)$ & 217 (dec.) & $\mathrm{MeOH}$ & This work \\
\hline $3(\mathrm{hq}) \cdot 2\left(2,2^{\prime}-\mathrm{bpe}\right)$ & 119-128 (dec.) & $\mathrm{MeOH}$ & This work \\
\hline$(\mathbf{h q}) \cdot\left(3,3^{\prime}\right.$-bpe $)$ & $167-171$ & $\mathrm{MeOH}$ & This work \\
\hline$(\mathbf{h q}) \cdot\left(4,4^{\prime}\right.$-bpe) $(730431)$ & 224-225 (dec.) & $\mathrm{MeOH}$ & This work \\
\hline
\end{tabular}


Table S10. Melting point data for X-bonded cocrystals and coformers thereof. For previously reported compounds, the CCDC deposition numbers are given in parentheses following the structural formula.

\begin{tabular}{|c|c|c|c|}
\hline Compound & $\mathbf{m p} /{ }^{\circ} \mathrm{C}$ & Solvent & Reference \\
\hline 1,2-di-I-tFb (875313) & $49-50$ & $\mathrm{CCl}_{4}$ & Chem. Eur. J. 2003, 9, 3974-3983 \\
\hline 1,3-di-I-tFb & Liquid at SATP & - & - \\
\hline 1,4-di-I-tFb (1317872) & $108-110$ & $\mathrm{CCl}_{4}$ & Chem. Eur. J. 2003, 9, 3974-3983 \\
\hline 2,2'-bpe & $119-120$ & $\mathrm{PE}$ & J. Org. Chem. 1982, 47, 3497-3503 \\
\hline 3,3'-bpe & 90 & $\mathrm{PhH}$ & ChemPhysChem 2020, 21, 154-163 \\
\hline \multirow[t]{3}{*}{ 4,4'-bpe } & $150-153$ & $\mathrm{CHCl}_{3}$ & Chem. Eur. J. 2003, 9, 3974-3983 \\
\hline & $149-150$ & $\mathrm{PhH}$ & Chem. Pharm. Bull. 1967, 15, 511-514 \\
\hline & $151-152$ & $\mathrm{PhH}$ & $\begin{array}{l}\text { J. Am. Chem. Soc. 1948, 70, 2330- } \\
2333\end{array}$ \\
\hline 2(1,2-di-I-tFb)·(2,2'-bpe) (1860151) & $86-88$ & $\mathrm{CHCl}_{3}$ & ChemPhysChem 2020, 21, 154-163 \\
\hline 2(1,2-di-I-tFb)·(3,3’-bpe) (1864394) & $123-125$ & $\mathrm{CHCl}_{3}$ & ChemPhysChem 2020, 21, 154-163 \\
\hline (1,2-di-I-tFb) (4,4'-bpe) (1863428) & 147-148 (dec.) & $\mathrm{CHCl}_{3}$ & ChemPhysChem 2020, 21, 154-163 \\
\hline $\begin{array}{l}\text { 2(1,3-di-I-tFb)·2(2,2'-bpe) } \\
(1940759)\end{array}$ & $90-91$ & $\mathrm{CHCl}_{3}$ & Molecules 2020, 25, 907-920 \\
\hline (1,3-di-I-tFb) (3,3'-bpe) & $102-105$ (dec.) & $\mathrm{CHCl}_{3}$ & This work \\
\hline$(1,3-d i-I-t F b) \cdot(4,4 ’-b p e)$ & 159 (dec.) & $\mathrm{CHCl}_{3}$ & This work \\
\hline (1,4-di-I-tFb) (2,2'-bpe) (1940762) & 155-156 (dec.) & $\mathrm{CHCl}_{3}$ & Molecules 2020, 25, 907-920 \\
\hline$(1,4-d i-I-t F b) \cdot(3,3 ’-b p e)$ & 138-140 (dec.) & $\mathrm{CHCl}_{3}$ & This work \\
\hline (1,4-di-I-tFb) (4,4'-bpe) (161324) & $>260$ (dec.) & $\mathrm{CHCl}_{3}$ & This work \\
\hline
\end{tabular}

\section{S5. References}

(1) a) Gordillo, A.; Ortuño, M. A.; López-Mardomingo, C.; Lledós, A.; Ujaque, G.; de Jesús, E. Mechanistic Studies on the Pd-Catalyzed Vinylation of Aryl Halides with Vinylalkoxysilanes in Water: The Effect of the Solvent and NaOH Promoter. J. Am. Chem. Soc. 2013, 135, 13749-13763; b) Gordillo, A.; de Jesús, E.; López-Mardomingo, C. Consecutive Palladium-Catalyzed Hiyama-Heck Reactions in Aqueous Media Under Ligand-Free Conditions. Chem. Commun. 2007, 4056-4058; c) Quentin, J.; MacGillivray, L. R. Halogen versus Hydrogen Bonding in Binary Cocrystals: Novel Conformation a Coformer with [2+2] Photoreactivity of Criss-Crossed C=C Bonds. ChemPhysChem 2020, 21, 154-163.

(2) Macrae, C. F.; Bruno, I. J.; Chisholm, J. A.; Edgington, P. R.; McCabe, P.; Pidcock, E.; Rodriguez-Monge, L.; Taylor, R.; van de Streek, J.; Wood, P. A. Mercury CSD 2.0 - New Features for the Visualization and Investigation of Crystal Structures. J. Appl. Cryst. 2008, 41, 466-470.

(3) Otwinowski, Z.; Minor, W. Processing of X-ray Diffraction Data Collected in Oscillation Mode. Methods Enzymol. 1997, 276, 307-326.

(4) Sheldrick, G. M. SHELXT - Integrated Space-Group and Crystal-Structure Determination. Acta Cryst. 2015, A71, 3-8.

(5) Sheldrick, G. M. Crystal Structure Refinement with SHELXL. Acta Cryst. 2015, C71, 3-8.

(6) Dolomanov, O. V.; Bourhis, L. J.; Gildea, R. J.; Howard, J. A. K.; Puschmann, H. OLEX2: a complete structure solution, refinement and analysis program. J. Appl. Cryst. 2009, 42, 339-341.

(7) Spek, A. L. Structure Validation in Chemical Crystallography. Acta Cryst. 2009, D65, 148-155. 